\title{
SIMPLE NORMAL CROSSING FANO VARIETIES AND LOG FANO MANIFOLDS
}

\author{
KENTO FUJITA
}

\begin{abstract}
A projective $\log$ variety $(X, D)$ is called a log Fano manifold if $X$ is smooth and if $D$ is a reduced simple normal crossing divisor on $X$ with $-\left(K_{X}+D\right)$ ample. The $n$-dimensional $\log$ Fano manifolds $(X, D)$ with nonzero $D$ are classified in this article when the log Fano index $r$ of $(X, D)$ satisfies either $r \geq n / 2$ with $\rho(X) \geq 2$ or $r \geq n-2$. This result is a partial generalization of the classification of logarithmic Fano 3-folds by Maeda.
\end{abstract}

\section{$\S 1$. Introduction}

As is well known, Fano manifolds play an essential role in various situations. Fano manifolds have been classified up to dimension 3. It is also known that the anticanonical degree of $n$-dimensional Fano manifolds is bounded for an arbitrary $n$. For a Fano manifold $X$, the Fano index is, by definition, the largest positive integer $r=r(X)$ such that $-K_{X}$ is $r$ times a Cartier divisor, and the Fano pseudoindex is the minimum $\iota=\iota(X)$ of the intersection number $\iota$ of $-K_{X}$ with a rational curve. We note that $n$-dimensional Fano manifolds $X$ with $r(X) \geq n-2$ have been classified (see [KO], [Ft2], [I], [MMu], [Mu2], [W1], [W2], and [W4]).

The Mukai conjecture [Mu1, Conjecture 4] asserts that $\rho(X)(r(X)-1) \leq$ $n$, and the generalized Mukai conjecture asserts that $\rho(X)(\iota(X)-1) \leq n$ for any Fano manifold $X$, where $\rho(X)$ is the Picard number of $X$. The generalized Mukai conjecture is important in the classification theory of Fano manifolds and is still open even now except for the case $n \leq 5$ or $\rho(X) \leq 3$ (see [NO] and references therein). One of the most significant results related to the Mukai conjecture is due to Wiśniewski ([W2], [W4]); he has classified $n$-dimensional Fano manifolds with $r(X)>n / 2$ and $\rho(X) \geq 2$.

Received June 11, 2012. Revised March 19, 2013. Accepted May 14, 2013.

First published online February 24, 2014.

2010 Mathematics Subject Classification. Primary 14J45; Secondary 14E30.

The author's work was partially supported by a Japan Society for the Promotion of Science Fellowship for Young Scientists. 
In this article, we consider simple normal crossing (SNC) Fano varieties - that is, projective simple normal crossing varieties whose dualizing sheaves are dual of ample invertible sheaves. In order to investigate SNC Fano varieties, it is natural to consider all of their irreducible components with the conductor divisors. The component with the conductor was considered by Maeda [M] in his study of logarithmic Fano varieties, that is, what we call a $\log$ Fano manifold in this article, which is a pair $(X, D)$ consisting of a smooth projective variety $X$ and a reduced SNC divisor $D$ on $X$ with $-\left(K_{X}+D\right)$ ample. Maeda classified such pairs with $\operatorname{dim} X \leq 3$ and pointed out that the degree $\left(-\left(K_{X}+D\right)^{\cdot n}\right)$ is unbounded for log Fano manifolds $(X, D)$ of fixed dimension $n \geq 3$.

We introduce the SNC Fano indices (resp., log Fano indices) and SNC Fano pseudoindices (resp., log Fano pseudoindices) for SNC Fano varieties (resp., for log Fano manifolds) similarly to the case of Fano manifolds (see Definition 2.8). It is expected that SNC Fano varieties with large SNC Fano indices have analogous applications (see, e.g., [Kol3]).

The following is the main idea to investigate $n$-dimensional log Fano manifolds $(X, D)$ with $D \neq 0$. Consider the contraction morphism associated to an extremal ray intersecting $D$ positively. Moreover, $D$ is an $(n-1)$ dimensional SNC Fano variety with $r(X, D) \mid r(D)$ (i.e., $r(X, D)$ divides $r(D))$ and $\iota(D) \geq \iota(X, D)$. Hence, we can use inductive arguments.

This article has the following organization. Section 2 is a preliminary section. We define SNC Fano varieties and log Fano manifolds in Sections 2.1 and 2.2 and we give some properties in Section 2.3. We show results on bundle structures (Section 2.4), extremal contractions (Section 2.5), and the special projective bundles, the so-called rational scrolls (Section 2.6). In Section 3, we give various examples of log Fano manifolds with large $\log$ Fano indices, which occur in the theorems in Section 4. In Section 4, we state the main results of this article. In Section 4.1 , we treat an $n$ dimensional $\log$ Fano manifold $(X, D)$ with $D \neq 0$ such that $r(X, D)=n-1$ (Proposition 4.2). The main purpose of this article, which we discuss in Section 4.2 , is to classify $n$-dimensional log Fano manifolds $(X, D)$ with $D \neq 0, r(X, D) \geq n / 2$, and $\rho(X) \geq 2$ (Theorems 4.3 and 4.5 ), which is a log version of the treatment of the Mukai conjecture by Wiśniewski ([W2], [W4]). We prove Theorem 4.5 in Section 5. Wiśniewski argued the case $r(X)>n / 2$, and we treat the case $r(X, D) \geq n / 2$ with $D \neq 0$. We remark that we do not treat Maeda's case $n=3$ and $r(X, D)=1$; some of the 
techniques of the proof are similar to Maeda's, but the objects of our study are completely different.

Theorem 1.1 (see Theorem 4.3). If $(X, D)$ is an $n$-dimensional log Fano manifold with $\iota:=\iota(X, D)>n / 2, D \neq 0$, and $\rho(X) \geq 2$, then $n=2 \iota-1$ and $(X, D) \simeq\left(\mathbb{P}\left[\mathbb{P}^{\iota-1} ; 0^{\iota}, m\right], \mathbb{P}^{\iota-1} \times \mathbb{P}^{\iota-1}\right)$ with $m \geq 0$, where the embedding $D \subset X$ is the canonical embedding $\mathbb{P}\left[\mathbb{P}^{\iota-1} ; 0^{\iota}\right] \subset$ can $\mathbb{P}\left[\mathbb{P}^{\iota-1} ; 0^{\iota}, m\right]$. (This is exactly the case in Example $O$ in Section 3.)

Theorem 1.2 (see Main Theorem 4.5). Let $(X, D)$ be a $2 r$-dimensional log Fano manifold with $r(X, D)=r \geq 2, D \neq 0$, and $\rho(X) \geq 2$. Then $(X, D)$ is in exactly one of Examples $I-X I$.

As a consequence of Theorems 4.3 and 4.5, together with Maeda's result, we have classified $n$-dimensional $\log$ Fano manifolds $(X, D)$ with $r(X, D) \geq$ $n-2$ and $D \neq 0$, which we discuss in Section 4.3 (see Corollary 4.6).

\section{Notation and terminology}

We always work in the category of algebraic (separated and finite type) schemes over a fixed algebraically closed field $\mathbb{k}$ of characteristic 0 . A variety means a connected and reduced algebraic scheme. (For the theory of extremal contraction, we refer readers to $[\mathrm{KolM}]$.) For a complete variety $X$, the Picard number of $X$ is denoted by $\rho(X)$. For a smooth projective variety $X$, we define $\operatorname{Eff}(X)$ (resp., $\operatorname{Nef}(X)$ ) to be the effective (resp., nef) cone, which is defined as the cone in $\mathrm{N}^{1}(X)$ spanned by the classes of effective (resp., nef) divisors on $X$. For a smooth projective variety $X$, let $\mathrm{NE}(X)$ be the cone in $\mathrm{N}_{1}(X)$ spanned by effective 1-cycles on $X$, and let $\overline{\mathrm{NE}}(X)$ be the closure of $\mathrm{NE}(X)$ in $\mathrm{N}_{1}(X)$. For a smooth projective variety $X$ and a $K_{X}$-negative extremal ray $R \subset \overline{\mathrm{NE}}(X)$, let $l(R):=\min \left\{\left(-K_{X} \cdot C\right) \mid\right.$ $C$ is a rational curve with $[C] \in R\}$. This is called the length $l(R)$ of $R$. A rational curve $C \subset X$ with $[C] \in R$ and $\left(-K_{X} \cdot C\right)=l(R)$ is called $a$ minimal rational curve of $R$.

For a morphism of algebraic schemes $f: X \rightarrow Y$, we define the exceptional locus $\operatorname{Exc}(f)$ of $f$ by $\operatorname{Exc}(f):=\{x \in X \mid f$ is not an isomorphism around $x\}$.

For a complete variety $X$, an invertible sheaf $\mathcal{L}$ on $X$, and $i \in \mathbb{Z}_{\geq 0}$, $\operatorname{dim}_{\mathbb{k}} H^{i}(X, \mathcal{L})$ is denoted by $h^{i}(X, \mathcal{L})$ (or simply by $h^{i}(X, L)$ if $\mathcal{L}=\mathcal{O}_{X}(L)$ ), where $\mathbb{Z}_{\geq 0}:=\{r \in \mathbb{Z} \mid r \geq 0\}$.

For algebraic schemes (or coherent sheaves on a fixed algebraic scheme) $X_{1}, \ldots, X_{m}$, the projection is denoted by $p_{i_{1}, \ldots, i_{k}}: \prod_{i=1}^{m} X_{i} \rightarrow \prod_{j=1}^{k} X_{i_{j}}$ for any $1 \leq i_{1}<\cdots<i_{k} \leq m$. 
For an algebraic scheme $X$ and a locally free sheaf of finite rank $\mathcal{E}$ on $X$, let $\mathbb{P}_{X}(\mathcal{E})$ be the projectivization of $\mathcal{E}$ in the sense of Grothendieck, and let $\mathcal{O}_{\mathbb{P}}(1)$ be the tautological invertible sheaf. We usually denote the projection by $p: \mathbb{P}_{X}(\mathcal{E}) \rightarrow X$. For locally free sheaves $\mathcal{E}_{1}, \ldots, \mathcal{E}_{m}$ of finite rank on $X$ and $1 \leq i_{1}<\cdots<i_{k} \leq m$, we sometimes denote the embedding obtained by the natural projection $p_{i_{1}, \ldots, i_{k}}: \bigoplus_{i=1}^{m} \mathcal{E}_{i} \rightarrow \bigoplus_{j=1}^{k} \mathcal{E}_{i_{j}}$ by

$$
\mathbb{P}_{X}\left(\bigoplus_{j=1}^{k} \mathcal{E}_{i_{j}}\right) \subset_{\text {can }} \mathbb{P}_{X}\left(\bigoplus_{i=1}^{m} \mathcal{E}_{i}\right)
$$

and we call this embedding the canonical embedding.

The symbol $\mathbb{Q}^{n}$ (resp., $\mathcal{Q}^{n}$ ) means a smooth (resp., possibly nonsmooth or reducible) hyperquadric in $\mathbb{P}^{n+1}$ for $n \geq 2$. We write $\mathcal{O}_{\mathbb{Q}^{n}}(1)\left(\right.$ resp., $\left.\mathcal{O}_{\mathcal{Q}^{n}}(1)\right)$ for the invertible sheaf which is the restriction of $\mathcal{O}_{\mathbb{P} n+1}(1)$ under the natural embedding. We sometimes write $\mathcal{O}(m)$ instead of $\mathcal{O}_{\mathbb{Q}^{n}}(m)$ (or $\mathcal{O}_{\mathcal{Q}^{n}}(m)$, $\left.\mathcal{O}_{\mathbb{P}^{n}}(m)\right)$ for simplicity.

For an irreducible projective variety $V$ with $\operatorname{Pic}(V)=\mathbb{Z}$, the ample generator $\mathcal{O}_{V}(1)$ of $\operatorname{Pic}(V)$, a nonnegative integer $t$, and integers $a_{0}, \ldots, a_{t}$, we denote the projective space bundle

$$
\mathbb{P}_{V}\left(\mathcal{O}_{V}\left(a_{0}\right) \oplus \cdots \oplus \mathcal{O}_{V}\left(a_{t}\right)\right)
$$

by $\mathbb{P}\left[V ; a_{0}, \ldots, a_{t}\right]$ for simplicity. (We often denote

$$
\mathbb{P}[V ; \underbrace{b_{0}, \ldots, b_{0}}_{n_{0} \text { times }}, \ldots, \underbrace{b_{u}, \ldots, b_{u}}_{n_{u} \text { times }}]
$$

by $\mathbb{P}\left[V ; b_{0}^{n_{0}}, \ldots, b_{u}^{n_{u}}\right]$ for any integers $b_{0}, \ldots, b_{u}$ and positive integers $n_{0}$, $\ldots, n_{u}$.) We also denote by $\mathcal{O}(m ; n)$ the invertible sheaf

$$
p^{*} \mathcal{O}_{V}(m) \otimes \mathcal{O}_{\mathbb{P}}(n) \quad \text { on } \mathbb{P}\left[V ; a_{0}, \ldots, a_{t}\right]
$$

for any integers $m$ and $n$, where $p: \mathbb{P}\left[V ; a_{0}, \ldots, a_{t}\right] \rightarrow V$ is the projection and $\mathcal{O}_{\mathbb{P}}(1)$ is the tautological invertible sheaf with respect to $p$. For any $0 \leq i_{1}<\cdots<i_{k} \leq t$, we denote the canonical embedding

$$
\mathbb{P}_{V}\left(\mathcal{O}_{V}\left(a_{i_{1}}\right) \oplus \cdots \oplus \mathcal{O}_{V}\left(a_{i_{k}}\right)\right) \subset \text { can } \mathbb{P}_{V}\left(\mathcal{O}_{V}\left(a_{0}\right) \oplus \cdots \oplus \mathcal{O}_{V}\left(a_{t}\right)\right)
$$

by $\mathbb{P}\left[V ; a_{i_{1}}, \ldots, a_{i_{k}}\right] \subset$ can $\mathbb{P}\left[V ; a_{0}, \ldots, a_{t}\right]$, and we call this the canonical embedding. 


\section{$\S 2$. Preliminaries}

\subsection{SNC varieties and log manifolds}

First, we define SNC varieties and log manifolds.

Definition 2.1. Let $X$ be a variety, and let $x \in X$ be a closed point. We say that $X$ has normal crossing singularity at $x$ if the completion of the local $\operatorname{ring} \mathcal{O}_{X, x}$ is isomorphic to $\mathbb{k}\left[\left[x_{1}, \ldots, x_{n+1}\right]\right] /\left(x_{1} \cdots x_{k}\right)$ for some $1 \leq k \leq n+1$.

\section{Definition 2.2.}

(1) An SNC variety is a variety $\mathcal{X}$ having normal crossing singularities at any closed points $x \in \mathcal{X}$, and each irreducible component of $\mathcal{X}$ is a smooth variety.

(2) A log manifold is a pair $(X, D)$ such that $X$ is a smooth variety and $D$ is an SNC divisor on $X$; that is, $D$ is a reduced divisor in $X$ which is an $\mathrm{SNC}$ variety.

Definition 2.3. Let $\mathcal{X}$ be an SNC variety with the irreducible decomposition $\mathcal{X}=\bigcup_{1 \leq i \leq m} X_{i}$. For any distinct $1 \leq i, j \leq m$, the intersection $X_{i} \cap X_{j}$ can be seen as a smooth divisor $D_{i j}$ in $X_{i}$. We define $D_{i}:=\sum_{j \neq i} D_{i j}$ and call it the conductor divisor in $X_{i}$ (with respect to $\mathcal{X}$ ). We often write that $\left(X_{i}, D_{i}\right) \subset \mathcal{X}$ is an irreducible component for the sake of simplicity. We also write $\mathcal{X}=\bigcup_{1 \leq i \leq m}\left(X_{i}, D_{i}\right)$.

Remark 2.4. If $\mathcal{X}$ is an SNC variety, then $\mathcal{X}$ has an invertible dualizing sheaf $\omega_{\mathcal{X}}$ since $\mathcal{X}$ has only Gorenstein singularities (see [Mat, Theorems 21.2(iii) and 21.3, Exercise 18.2]). Furthermore, if $(X, D) \subset \mathcal{X}$ is an irreducible component with its conductor divisor, then $(X, D)$ is a log manifold and $\left.\omega_{\mathcal{X}}\right|_{X} \simeq \mathcal{O}_{X}\left(K_{X}+D\right)$ by the adjunction formula.

Definition 2.5 ([F, Definition 2.10]). Let $\mathcal{X}$ be an SNC variety with the irreducible decomposition $\mathcal{X}=\bigcup_{1 \leq i \leq m} X_{i}$. A stratum of $\mathcal{X}$ is an irreducible component of $\bigcap_{i \in I} X_{i}$ with the reduced scheme structure for a subset $I \subset$ $\{1, \ldots, m\}$. A minimal stratum of $\mathcal{X}$ is a stratum of $\mathcal{X}$ which is a minimal in the set of strata of $\mathcal{X}$ under the partial order of the inclusion.

Now, we consider the descent of invertible sheaves.

Proposition 2.6. Let $\mathcal{X}$ be an $n$-dimensional $S N C$ variety with irreducible decomposition $\mathcal{X}=\bigcup_{i=1}^{m} X_{i}$, which has a unique minimal stratum. We also let $X_{i j}:=X_{i} \cap X_{j}$ (scheme theoretic intersection) for any $1 \leq i<$ 
$j \leq m$. Then we have an exact sequence

$$
0 \rightarrow \operatorname{Pic}(\mathcal{X}) \stackrel{\eta}{\rightarrow} \bigoplus_{i=1}^{m} \operatorname{Pic}\left(X_{i}\right) \stackrel{\mu}{\rightarrow} \bigoplus_{1 \leq i<j \leq m} \operatorname{Pic}\left(X_{i j}\right)
$$

where $\eta$ is the restriction homomorphism and where $\mu\left(\left(\mathcal{H}_{i}\right)_{i}\right):=\left(\left.\mathcal{H}_{i}\right|_{X_{i j}} \otimes\right.$ $\left.\left.\mathcal{H}_{j}^{\vee}\right|_{X_{i j}}\right)_{i<j}$.

Proof. Let $\mathcal{X}_{i}:=\bigcup_{j=1}^{i} X_{j} \subset \mathcal{X}$. Then both $\mathcal{X}_{i}$ and $\mathcal{X}_{i} \cap X_{i+1}$ are SNC varieties and have a unique minimal stratum. Since units of structure sheaves form an exact sequence

$$
1 \rightarrow \mathcal{O}_{\mathcal{X}_{i+1}}^{*} \rightarrow \mathcal{O}_{X_{i+1}}^{*} \times \mathcal{O}_{\mathcal{X}_{i}}^{*} \rightarrow \mathcal{O}_{\mathcal{X}_{i} \cap X_{i+1}}^{*} \rightarrow 1
$$

of sheaves of abelian groups, there is a long exact sequence

$$
\mathbb{k}^{*} \times \mathbb{k}^{*} \stackrel{v}{\rightarrow} \mathbb{k}^{*} \rightarrow \operatorname{Pic}\left(\mathcal{X}_{i+1}\right) \stackrel{\lambda}{\rightarrow} \operatorname{Pic}\left(X_{i+1}\right) \oplus \operatorname{Pic}\left(\mathcal{X}_{i}\right) \rightarrow \operatorname{Pic}\left(\mathcal{X}_{i} \cap X_{i+1}\right) .
$$

The map $\lambda$ above is injective since $v$ is surjective. In particular, $\eta$ is injective. It is obvious that $\mu \circ \eta=0$. Assume that $\left(\mathcal{H}_{i}\right)_{i} \in \bigoplus_{i=1}^{m} \operatorname{Pic}\left(X_{i}\right)$ satisfies $\mu\left(\left(\mathcal{H}_{i}\right)_{i}\right)=0$. We will show that there exist invertible sheaves $\mathcal{L}_{i} \in \operatorname{Pic}\left(\mathcal{X}_{i}\right)$ for all $1 \leq i \leq m$, which satisfy $\left.\mathcal{L}_{i}\right|_{X_{i}} \simeq \mathcal{H}_{i}$ and $\left.\mathcal{L}_{i}\right|_{\mathcal{X}_{i-1}} \simeq \mathcal{L}_{i-1}$ (if $i \geq 2$ ). If $i=1$, then $\mathcal{L}_{1}$ must be (isomorphic to) $\mathcal{H}_{1}$. Assume that we have constructed $\mathcal{L}_{1}, \ldots, \mathcal{L}_{i}$. Since

$$
\operatorname{Pic}\left(\mathcal{X}_{i+1}\right) \rightarrow \operatorname{Pic}\left(X_{i+1}\right) \oplus \operatorname{Pic}\left(\mathcal{X}_{i}\right) \rightarrow \operatorname{Pic}\left(\mathcal{X}_{i} \cap X_{i+1}\right)
$$

is exact, it is enough to show that $\left.\left.\mathcal{L}_{i}\right|_{\mathcal{X}_{i} \cap X_{i+1}} \simeq \mathcal{H}_{i+1}\right|_{\mathcal{X}_{i} \cap X_{i+1}}$ to construct $\mathcal{L}_{i+1}$. We know that the map $\kappa: \operatorname{Pic}\left(\mathcal{X}_{i} \cap X_{i+1}\right) \rightarrow \bigoplus_{j=1}^{i} \operatorname{Pic}\left(X_{j, i+1}\right)$ is injective since $\mathcal{X}_{i} \cap X_{i+1}$ has a unique minimal stratum. Both $\left.\mathcal{L}_{i}\right|_{\mathcal{X}_{i} \cap X_{i+1}}$ and $\left.\mathcal{H}_{i+1}\right|_{\mathcal{X}_{i} \cap X_{i+1}}$ map $\kappa$ to $\left(\left.\mathcal{H}_{j}\right|_{X_{j, i+1}}\right)_{j}$; thus, we can construct $\mathcal{L}_{i+1}$. Hence, we construct $\mathcal{L}_{i} \in \operatorname{Pic}\left(\mathcal{X}_{i}\right)$ for all $1 \leq i \leq m$ by induction. For any $1 \leq i \leq m$, $\left.\mathcal{L}_{m}\right|_{X_{i}} \simeq \mathcal{H}_{i}$ holds by construction. Thus, $\eta\left(\mathcal{L}_{m}\right)=\left(\mathcal{H}_{i}\right)_{i}$.

\subsection{SNC Fano varieties and log Fano manifolds}

Definition 2.7. We have the following.

(1) A projective SNC variety $\mathcal{X}$ is said to be an SNC Fano variety if the dual of the dualizing sheaf $\omega_{\mathcal{X}}^{\vee}$ is ample.

(2) A projective $\log$ manifold $(X, D)$ is said to be a log Fano manifold if $-\left(K_{X}+D\right)$ is ample. 
We define the index and pseudoindex of an SNC Fano variety and also of a log Fano manifold, whose notion is essential to our article.

Definition 2.8. Let $\mathcal{X}$ be an SNC Fano variety. We define the SNC Fano index $r(\mathcal{X})$ (resp., the $S N C$ Fano pseudoindex $\iota(\mathcal{X})$ ) of $\mathcal{X}$ as $\max \left\{r \in \mathbb{Z}_{>0} \mid\right.$ $\omega_{\mathcal{X}}^{\vee} \simeq \mathcal{L}^{\otimes r}$ for some $\left.\mathcal{L} \in \operatorname{Pic}(\mathcal{X})\right\}$ (resp., $\min \left\{\operatorname{deg}_{C}\left(\left.\omega_{\mathcal{X}}^{\vee}\right|_{C}\right) \mid C \subset \mathcal{X}\right.$ rational curve $\}$ ). For a log Fano manifold $(X, D)$, the log Fano index $r(X, D)$ and the $\log$ Fano pseudoindex $\iota(X, D)$ are similarly defined by replacing $\omega_{\mathcal{X}}$ by $\mathcal{O}_{X}\left(K_{X}+D\right)$.

Remark 2.9. For an SNC Fano variety $\mathcal{X}$, we have $r(\mathcal{X}) \mid \iota(\mathcal{X})$. For a log Fano manifold $(X, D)$, we have $r(X, D) \mid \iota(X, D)$.

Remark 2.10. Let $\mathcal{X}$ be an $n$-dimensional SNC Fano variety, and let $(X, D) \subset \mathcal{X}$ be an irreducible component with its conductor. Then $(X, D)$ is an $n$-dimensional log Fano manifold such that $r(\mathcal{X}) \mid r(X, D)$ and $\iota(X, D) \geq$ $\iota(\mathcal{X})$ by Remark 2.4 .

\subsection{First properties of log Fano manifolds}

Theorem 2.11 ([KolM, Theorem 3.35]). Let $(X, D)$ be a log Fano manifold. Then $\mathrm{NE}(X)$ is spanned by a finite number of extremal rays. Furthermore, for any extremal ray $R \subset \mathrm{NE}(X)$, the ray $R$ is spanned by a class of rational curve $C$ on $X$, and there exists a contraction morphism cont $_{R}: X \rightarrow Y$ associated to $R$. Moreover, if $\mathcal{L} \in \operatorname{Pic}(X)$ satisfies $(\mathcal{L} \cdot C)=0$, then there exists $\mathcal{M} \in \operatorname{Pic}(Y)$ such that $\operatorname{cont}_{R}^{*} \mathcal{M} \simeq \mathcal{L}$.

Lemma 2.12 ([M, Corollary 2.2, Lemma 2.3]). Let $(X, D)$ be a log Fano manifold. Then $\operatorname{Pic}(X)$ is torsion-free and is isomorphic to $H^{2}\left(X^{\text {an }} ; \mathbb{Z}\right)$ if $\mathbb{k}=\mathbb{C}$.

Proposition 2.13. Let $(X, D)$ be a log Fano manifold with $\rho(X)=1$ and $D \neq 0$. Then $X$ is a Fano manifold such that $r(X)>r(X, D)$ and $\iota(X)>\iota(X, D)$.

TheOREM 2.14 ([M, Lemma 2.4]).

(1) Let $(X, D)$ be a log Fano manifold. Then $D$ is a (connected) SNC Fano variety such that $r(X, D) \mid r(D)$ and $\iota(D) \geq \iota(X, D)$ hold.

(2) Let $\mathcal{X}$ be an $S N C$ Fano variety. Then there is a unique minimal stratum of $\mathcal{X}$. In particular, any two irreducible components of $\mathcal{X}$ intersect each other. 
Proof. (1) We know that $D$ is connected by [M, Lemma 2.4(a)]. In modern terms, the connectedness of $D$ is obtained by Shokurov's connectedness theorem [Kol1, Theorem 17.4]. The other assertions follow from adjunction.

(2) We can prove Theorem 2.14 by using the same idea in [M, Lemma $\left.2.4\left(\mathrm{a}^{\prime}\right)\right]$. We remark that this is directly shown by $[\mathrm{A}$, Theorem $6.6(\mathrm{ii})]$ and [F, Theorem 3.47(ii)].

Now, we give the theorem that combines log Fano manifolds into an SNC Fano variety. By Theorem 2.14(2), for an SNC Fano variety $\mathcal{X}=$ $\bigcup_{i=1}^{m}\left(X_{i}, D_{i}\right)$, any two components $X_{i}, X_{j}$ intersect each other. Thus, there exist isomorphisms $\phi_{i j}: D_{i j} \rightarrow D_{j i}$ for all distinct $i, j$ such that $\phi_{j i}=\phi_{i j}^{-1}$ and $\left.\left.\phi_{j k}\right|_{D_{j i} \cap D_{j k}} \circ \phi_{i j}\right|_{D_{i j} \cap D_{i k}}=\left.\phi_{i k}\right|_{D_{i j} \cap D_{i k}}$ hold. Conversely, we have the following result. The proof follows from Kollár's gluing theory [Kol2, Theorem 23, Section 3] and from Proposition 2.6, Lemma 2.12, and Theorem 2.14(2).

Theorem 2.15. Fix $n, r, m \in \mathbb{Z}_{>0}$. Let $\left(X_{i}, D_{i}\right)$ be an $n$-dimensional log Fano manifold such that $r \mid r\left(X_{i}, D_{i}\right)$ for any $1 \leq i \leq m$. Assume that the irreducible decomposition is written as $D_{i}=\sum_{j \neq i, 1 \leq j \leq m} D_{i j}$ for any $1 \leq i \leq m$ and that there exist isomorphisms $\phi_{i j}: D_{i j} \rightarrow D_{j i}$ for all distinct $1 \leq i, j \leq m$ which satisfy

(1) $\phi_{j i}=\phi_{i j}^{-1}$ for distinct $i, j$,

(2) $\phi_{i j}\left(D_{i j} \cap D_{i k}\right)=D_{j i} \cap D_{j k}$ and $\left.\phi_{j k} \circ \phi_{i j}\right|_{D_{i j} \cap D_{i k}}=\left.\phi_{i k}\right|_{D_{i j} \cap D_{i k}}$ for distinct $i, j, k$.

Then there exists an $n$-dimensional SNC Fano variety $\mathcal{X}$ such that $r$ $r(\mathcal{X})$ whose irreducible decomposition can be written as $\mathcal{X}=\bigcup_{i=1}^{m}\left(X_{i}, D_{i}\right)$.

\subsection{Bundles and subbundles}

In this section, we recall some bundle structures. The following lemma is well known.

Lemma 2.16. Let $X$ be an irreducible variety, let $D \subset X$ be an effective Cartier divisor, and let $c$ be a nonnegative integer. Let $\pi: X \rightarrow Y$ be a $\mathbb{P}^{c}$ bundle such that $\left.\pi\right|_{D}: D \rightarrow Y$ is a $\mathbb{P}^{c-1}$-subbundle. That is, $\pi$ is a proper and smooth morphism such that $\pi^{-1}(y) \simeq \mathbb{P}^{c}$ and such that $\left(\left.\pi\right|_{D}\right)^{-1}(y)$ is isomorphic to a hyperplane section under this isomorphism for any closed point $y \in Y$. Then $X$ is isomorphic to $\mathbb{P}_{Y}\left(\pi_{*} \mathcal{O}_{X}(D)\right)$ over $Y$. Moreover, under the isomorphism, $D$ is isomorphic to $\mathbb{P}_{Y}\left(\left(\left.\pi\right|_{D}\right)_{*} \mathcal{N}_{D / X}\right)$ and the embedding is induced by the natural surjection $\pi_{*} \mathcal{O}_{X}(D) \rightarrow\left(\left.\pi\right|_{D}\right)_{*} \mathcal{N}_{D / X}$, where $\mathcal{N}_{D / X}$ is 
the normal sheaf $\mathcal{O}_{D}(D)$. Furthermore, we have $D \in\left|\mathcal{O}_{\mathbb{P}}(1)\right|$ under the isomorphism, where $\mathcal{O}_{\mathbb{P}}(1)$ is the tautological invertible sheaf on $\mathbb{P}_{Y}\left(\pi_{*} \mathcal{O}_{X}(D)\right)$.

Next, we consider $\mathcal{Q}^{c+1}$-bundles and $\mathcal{Q}^{c}$-subbundles.

Definition 2.17. Let $\pi: X \rightarrow Y$ be a morphism between irreducible varieties, and let $c$ be a positive integer. We say that $\pi: X \rightarrow Y$ is a $\mathcal{Q}^{c+1}$-bundle if $\pi$ is a proper, flat morphism and if $\pi^{-1}(y)$ is (scheme theoretically) isomorphic to a hyperquadric in $\mathbb{P}^{c+2}$. For a $\mathcal{Q}^{c+1}$-bundle $\pi: X \rightarrow Y$ and an effective Cartier divisor $D$ on $X$, we say that $\left.\pi\right|_{D}: D \rightarrow Y$ is a $\mathcal{Q}^{c}$-subbundle of $\pi$ if $\left(\left.\pi\right|_{D}\right)^{-1}(y)$ is isomorphic to a hyperplane section under the isomorphism $\pi^{-1}(y) \simeq \mathcal{Q}^{c+1}$ for any closed point $y \in Y$. We note that the morphisms $\pi$ and $\left.\pi\right|_{D}$ need not be smooth. (That is why we use the symbol $\mathcal{Q}$, not $\mathbb{Q}$.)

The following lemma is proved similarly to Lemma 2.16 and is well known.

LEMma 2.18. Let $X$ be an irreducible variety, let $D \subset X$ be an effective Cartier divisor, let $Y$ be a smooth variety, and let $c$ be a positive integer. Suppose that $\pi: X \rightarrow Y$ is a $\mathcal{Q}^{c+1}$-bundle and that $\left.\pi\right|_{D}: D \rightarrow Y$ is a $\mathcal{Q}^{c}$ subbundle. Then we have the following.

(i) The natural sequence $0 \rightarrow \mathcal{O}_{Y} \rightarrow \pi_{*} \mathcal{O}_{X}(D) \rightarrow\left(\left.\pi\right|_{D}\right)_{*} \mathcal{N}_{D / X} \rightarrow 0$ is exact.

(ii) Both $\pi_{*} \mathcal{O}_{X}(D)$ and $\left(\left.\pi\right|_{D}\right)_{*} \mathcal{N}_{D / X}$ are locally free, of rank $c+3$ and $c+2$, respectively. In particular, $P:=\mathbb{P}_{Y}\left(\pi_{*} \mathcal{O}_{X}(D)\right)$ is a $\mathbb{P}^{c+2}$-bundle over $Y$, and $H:=\mathbb{P}_{Y}\left(\left(\left.\pi\right|_{D}\right)_{*} \mathcal{N}_{D / X}\right)$ is a $\mathbb{P}^{c+1}$-subbundle.

(iii) The natural homomorphism $\pi^{*} \pi_{*} \mathcal{O}_{X}(D) \rightarrow \mathcal{O}_{X}(D)$ is surjective, and it induces a relative quadric embedding $X \hookrightarrow P$ over $Y$.

(iv) The divisor $D$ is isomorphic to the complete intersection $X \cap H$ in $P$ under these embeddings.

Lemma 2.19. Let $X$ be an irreducible variety such that $h^{1}\left(X, \mathcal{O}_{X}\right)=0$.

(1) Let $c \in \mathbb{Z}_{\geq 0}$ and $p_{1}: X \times \mathbb{P}^{c} \rightarrow X, p_{2}: X \times \mathbb{P}^{c} \rightarrow \mathbb{P}^{c}$ be the projections. Then $\left(p_{1}\right)_{*}\left(p_{2}^{*} \mathcal{O}_{\mathbb{P}}(1)\right) \simeq \mathcal{O}_{X}^{\oplus c+1}$.

(2) Let $c \geq 2$ and $p_{1}: X \times \mathbb{Q}^{c} \rightarrow X, p_{2}: X \times \mathbb{Q}^{c} \rightarrow \mathbb{Q}^{c}$ be the projections. Then $\left(p_{1}\right)_{*}\left(p_{2}^{*} \mathcal{O}_{\mathbb{Q}^{c}}(1)\right) \simeq \mathcal{O}_{X}^{\oplus c+2}$.

Proof. We prove both assertions by induction on $c$.

(1) The case $c=0$ is trivial. We assume that the assertion holds for the case $c-1$. There is the canonical exact sequence

$$
0 \rightarrow \mathcal{O}_{X \times \mathbb{P}^{c}} \rightarrow p_{2}^{*} \mathcal{O}_{\mathbb{P}^{c}}(1) \rightarrow\left(\left.p_{2}\right|_{X \times \mathbb{P}^{c-1}}\right)^{*} \mathcal{O}_{\mathbb{P}^{c-1}}(1) \rightarrow 0
$$


After taking $\left(p_{1}\right)_{*}$, the sequence

$$
0 \rightarrow \mathcal{O}_{X} \rightarrow\left(p_{1}\right)_{*}\left(p_{2}^{*} \mathcal{O}_{\mathbb{P}^{c}}(1)\right) \rightarrow\left(\left.p_{1}\right|_{X \times \mathbb{P}^{c-1}}\right)_{*}\left(\left(\left.p_{2}\right|_{X \times \mathbb{P}^{c-1}}\right)^{*} \mathcal{O}_{\mathbb{P}^{c-1}}(1)\right) \rightarrow 0
$$

is exact. We note that $\left(\left.p_{1}\right|_{X \times \mathbb{P}^{c-1}}\right)_{*}\left(\left(\left.p_{2}\right|_{X \times \mathbb{P}^{c-1}}\right)^{*} \mathcal{O}_{\mathbb{P}^{c-1}}(1)\right) \simeq \mathcal{O}_{X}^{\oplus c}$ by the induction step. The sequence always splits since $h^{1}\left(X, \mathcal{O}_{X}\right)=0$. Thus, we have proved (1).

(2) The case $c=2$ is a direct consequence of $(1)$ since $\mathbb{Q}^{2}$ is isomorphic to $\mathbb{P}^{1} \times \mathbb{P}^{1}$. We assume that the assertion holds for the case $c-1$. There is the exact sequence

$$
0 \rightarrow \mathcal{O}_{X \times \mathbb{Q}^{c}} \rightarrow p_{2}^{*} \mathcal{O}_{\mathbb{Q}^{c}}(1) \rightarrow\left(\left.p_{2}\right|_{X \times \mathbb{Q}^{c-1}}\right)^{*} \mathcal{O}_{\mathbb{Q}^{c-1}}(1) \rightarrow 0
$$

After taking $\left(p_{1}\right)_{*}$, we have the splitting exact sequence

$$
0 \rightarrow \mathcal{O}_{X} \rightarrow\left(p_{1}\right)_{*}\left(p_{2}^{*} \mathcal{O}_{\mathbb{Q}^{c}}(1)\right) \rightarrow \mathcal{O}_{X}^{\oplus c+1} \rightarrow 0
$$

by repeating the argument in the proof of (1). Hence, we have proved (2).

\subsection{Facts on extremal contractions and its applications}

In this section, we describe the structure of the contraction morphism associated to a special ray.

We recall Wiśniewski's inequality, which plays an essential role in this section.

THEOREM 2.20 ([W3, p. 143]). Let $X$ be a smooth projective variety, and let $R \subset \overline{\mathrm{NE}}(X)$ be a $K_{X}$-negative extremal ray with the associated contraction morphism $\pi: X \rightarrow Y$. Then $\operatorname{dim} \operatorname{Exc}(\pi)+\operatorname{dim} F \geq \operatorname{dim} X+l(R)-1$ for any nontrivial fiber $F$ of $\pi$.

We give a criterion for a variety $X$ to have $\rho(X)=1$ using Theorem 2.20.

Lemma 2.21. Let $X$ be a smooth projective variety, let $D \subset X$ be a prime divisor, and let $R \subset \overline{\mathrm{NE}}(X)$ be a $K_{X}$-negative extremal ray with associated contraction morphism $\pi: X \rightarrow Y$ such that $(D \cdot R)>0$.

(1) If the restriction morphism $\left.\pi\right|_{D}: D \rightarrow \pi(D)$ is not birational, then $\pi$ is of fiber type; that is, $\operatorname{dim} Y<\operatorname{dim} X$ holds.

(2) If $l(R) \geq 3$, then $\left.\pi\right|_{D}: D \rightarrow Y$ is not a finite morphism. Furthermore, if $\rho(D)=1$ holds in addition, then $X$ is a Fano manifold with $\rho(X)=1$. 
Proof. (1) If $\pi$ is birational, then it is a divisorial contraction, and the exceptional divisor is exactly $D$, since $\left.\pi\right|_{D}: D \rightarrow \pi(D)$ is not birational. However, we get a contradiction since $(D \cdot R)>0$. Hence, $\pi$ is of fiber type.

(2) Let us choose an arbitrary nontrivial fiber $F$ of $\pi$. We have $D \cap F \neq \emptyset$ since $(D \cdot R)>0$. Then $\operatorname{dim}(F \cap D) \geq \operatorname{dim} F-1 \geq l(R)-2 \geq 1$ by Theorem 2.20. Hence, $F \cap D$ contains a curve. Now, we assume that $\rho(D)=1$. Then $\pi(D)$ must be a point since all curves in $D$ are numerically proportional. Therefore, $\pi$ is of fiber type by (1), and $Y$ must be a point since $(D \cdot R)>0$. In particular, $\rho(X)=1$. Thus, $X$ is a Fano manifold.

We show that there exists a special $K_{X}$-negative extremal ray for a log Fano manifold with nonzero boundary, which is essential for classifying some special log Fano manifolds.

Lemma 2.22. Let $(X, D)$ be a log Fano manifold with $r:=r(X, D)$, let $\iota:=\iota(X, D), L$ be a divisor on $X$ such that $-\left(K_{X}+D\right) \sim r L$ holds, and assume that $D \neq 0$. Then there exists an extremal ray $R \subset \mathrm{NE}(X)$ such that $(D \cdot R)>0$. Let $R$ be an extremal ray satisfying $(D \cdot R)>0$, and let $\pi: X \rightarrow$ $Y$ be the contraction morphism associated to $R$. Then $R$ is always $K_{X^{-}}$ negative and $l(R) \geq \iota+1$. Moreover, the restriction morphism $\left.\pi\right|_{D_{1}}: D_{1} \rightarrow$ $\pi\left(D_{1}\right)$ to its image is an algebraic fiber space, that is, $\left(\left.\pi\right|_{D_{1}}\right)_{*} \mathcal{O}_{D_{1}}=\mathcal{O}_{\pi\left(D_{1}\right)}$, for any irreducible component $D_{1} \subset D$. Furthermore, for a minimal rational curve $C \subset X$ of $R$, we have the following properties.

(1) If $l(R)=\iota+1$, then $(D \cdot C)=1$.

(2) If $l(R)=r+2$ and $r \geq 2$, then $(L \cdot C)=1$ and $(D \cdot C)=2$.

Proof. Such an extremal ray exists, since $D$ is a nonzero effective divisor and $\mathrm{NE}(X)$ is spanned by a finite number of extremal rays. Let $R \subset \mathrm{NE}(X)$ be an extremal ray such that $(D \cdot R)>0$. Then $R$ is $K_{X}$-negative since $\left(-K_{X} \cdot R\right)=\left(-\left(K_{X}+D\right) \cdot R\right)+(D \cdot R)>0$. To see that $\left.\pi\right|_{D_{1}}: D_{1} \rightarrow \pi\left(D_{1}\right)$ is an algebraic fiber space, it is enough to show that the homomorphism $\pi_{*} \mathcal{O}_{X} \rightarrow\left(\left.\pi\right|_{D_{1}}\right)_{*} \mathcal{O}_{D_{1}}$ is surjective. We know that $R^{1} \pi_{*} \mathcal{O}_{X}\left(-D_{1}\right)=0$ by a vanishing theorem (see, e.g., [F, Theorem 2.42]). Hence, $\left.\pi\right|_{D_{1}}: D_{1} \rightarrow \pi\left(D_{1}\right)$ is an algebraic fiber space. Let $C \subset X$ be a minimal rational curve of $R$. Then we have $l(R)=\left(-K_{X} \cdot C\right)=\left(-\left(K_{X}+D\right) \cdot C\right)+(D \cdot C) \geq \iota+1$. If $l(R)=\iota+1$, then the above inequality is in fact an equality. Hence, $(D \cdot C)=1$ holds. If $l(R)=r+2$ and if $r \geq 2$, then $r+2=l(R)=r(L \cdot C)+(D \cdot C) \geq r+1$. Therefore, $(L \cdot C)=1$ and $(D \cdot C)=2$ holds. 
Using Lemma 2.22, we show some delicate structure properties of certain log Fano manifolds.

Proposition 2.23. Let $(X, D)$ be a log Fano manifold with $r:=r(X, D)$, $\iota:=\iota(X, D)$, and $D \neq 0$. Pick an arbitrary extremal ray $R \subset \mathrm{NE}(X)$ such that $(D \cdot R)>0$, and let $\pi: X \rightarrow Y$ be the contraction morphism associated to $R$. Let $F$ be an arbitrary nontrivial fiber of $\pi$. Then $\operatorname{dim}(D \cap F) \geq \iota-1$ holds. Furthermore, we have the following results.

(i) If $\operatorname{dim}(D \cap F)=\iota-1$ for any nontrivial fiber $F$, then $\pi: X \rightarrow Y$ is a $\mathbb{P}^{\iota}$-bundle and $\left.\pi\right|_{D}: D \rightarrow Y$ is a $\mathbb{P}^{\iota-1}$-subbundle.

(ii) If $r \geq 2$ and there exists an irreducible component $D_{1}$ of $D$ such that $\operatorname{dim}\left(D_{1} \cap F\right)=r$ for any $F$, then one of the following holds:

(a) $Y$ is a smooth projective variety, and $\pi$ is the blowup along a smooth projective subvariety $W \subset Y$ of codimension $r+2$;

(b) $Y$ is smooth, $\pi: X \rightarrow Y$ is a $\mathcal{Q}^{r+1}$-bundle, and $\left.\pi\right|_{D_{1}}: D_{1} \rightarrow Y$ is a $\mathcal{Q}^{r}$-subbundle;

(c) $\pi: X \rightarrow Y$ is a $\mathbb{P}^{r+1}$-bundle, and $\left.\pi\right|_{D_{1}}: D_{1} \rightarrow Y$ is a $\mathbb{P}^{r}$-subbundle;

(d) $\pi_{*} \mathcal{O}_{X}(L)$ is locally free of rank $r+2$, where $L$ is any divisor on $X$ such that $-\left(K_{X}+D\right) \sim r L$. Furthermore, $\pi: X \rightarrow Y$ is isomorphic to the projection $p: \mathbb{P}_{Y}\left(\pi_{*} L\right) \rightarrow Y$, and $\left(\left.\pi\right|_{D_{1}}\right)^{-1}(y)$ is a hyperquadric section under the isomorphism $\pi^{-1}(y) \simeq \mathbb{P}^{r+1}$ for any closed point $y \in Y$. Moreover, $\pi_{*} \mathcal{O}_{X}(L) \simeq\left(\left.p\right|_{D_{1}}\right)_{*}\left(\left.\mathcal{O}_{\mathbb{P}}(1)\right|_{D_{1}}\right)$ under the isomorphism.

Proof. Let $L$ be a divisor on $X$ with $-\left(K_{X}+D\right) \sim r L$, and let $C$ be a minimal rational curve of $R$. We note that $D$ and $F$ intersect each other since $(D \cdot R)>0$. Hence,

$$
\begin{aligned}
\operatorname{dim}(D \cap F) & \geq \operatorname{dim} F-1 \geq \operatorname{dim} X-\operatorname{dim} \operatorname{Exc}(\pi)+l(R)-2 \\
& \geq l(R)-2 \geq \iota-1 \geq r-1
\end{aligned}
$$

by Theorem 2.20 and by Lemma 2.22 .

First, we consider the case (i). Then $\operatorname{dim} \operatorname{Exc}(\pi)=\operatorname{dim} X$ and $l(R)=\iota+1$. Hence, $\pi$ is of fiber type, all fibers of $\pi$ are of dimension $\iota$, and the equalities $(D \cdot C)=1$ and $\left(-K_{X} \cdot C\right)=\iota+1$ hold by Lemma 2.22 . Therefore, $\pi: X \rightarrow Y$ is a $\mathbb{P}^{\iota}$-bundle and $\left.\pi\right|_{D}: D \rightarrow Y$ is a $\mathbb{P}^{\iota-1}$-subbundle by [Ft1, Lemma 2.12].

Next, we consider case (ii). We first show that $\left(D_{1} \cdot R\right)>0$. If not, any nontrivial fiber $F$ is included in $D_{1}$. (In particular, $\pi$ is of birational type.) Then Theorem 2.20 and Lemma 2.22 show that $\operatorname{dim} \operatorname{Exc}(\pi)+r \geq \operatorname{dim} X+$ 
$l(R)-1 \geq \operatorname{dim} X+\iota$. Hence, $\pi$ is of fiber type. This leads to a contradiction. Consequently, we have $\left(D_{1} \cdot R\right)>0$.

We first assume that $\operatorname{dim} \operatorname{Exc}(\pi)<\operatorname{dim} X$. Then $\operatorname{dim} \operatorname{Exc}(\pi)=\operatorname{dim} X-1$ and $l(R)=r+1$ by substituting $D_{1}$ for (1). Hence, $\pi$ is a divisorial contraction such that $\operatorname{dim} F=r+1$ for any $F$, and the equality $(D \cdot C)=1$ holds by Lemma 2.22(1). Thus, $Y$ is a smooth projective variety and $\pi$ is the blowup whose center $W \subset Y$ is a smooth projective subvariety of codimension $r+2$ by $[$ AW, Theorem 4.1(iii)]. Therefore, condition (ii)(a) is satisfied in the case $\operatorname{dim} \operatorname{Exc}(\pi)<\operatorname{dim} X$.

Second, we assume that $\operatorname{dim} \operatorname{Exc}(\pi)=\operatorname{dim} X$, that is, that $\pi$ is of fiber type. We note that $l(R)=r+1$ or $r+2$ by $(1)$.

Assume that $\pi$ is of fiber type and that $l(R)=r+1$. Then $\operatorname{dim} F=r+1$ for any fiber, and the equalities $\left(D_{1} \cdot C\right)=1$ and $\left(-K_{X} \cdot C\right)=r+1$ hold by (1) and Lemma 2.22(1). Thus, $\pi_{*} \mathcal{O}_{X}\left(D_{1}\right)$ is locally free of rank $r+3$, and $X$ is embedded over $Y$ into $\mathbb{P}_{Y}\left(\pi_{*} \mathcal{O}_{X}\left(D_{1}\right)\right)$ as a divisor of relative degree 2 by $[\mathrm{ABW}$, Theorem B]. Therefore, condition (ii)(b) is satisfied in the case $\operatorname{dim} \operatorname{Exc}(\pi)=\operatorname{dim} X$ and $l(R)=r+1$.

Assume that $\pi$ is of fiber type and that $l(R)=r+2$. Then $(L \cdot C)=1$ and either $\left(D_{1} \cdot C\right)=1$ or 2 holds by Lemma 2.22. Thus, $\pi: X \rightarrow Y$ is isomorphic to the $\mathbb{P}^{r+1}$-bundle $\mathbb{P}_{Y}\left(\pi_{*} \mathcal{O}_{X}(L)\right)$ by [Ft1, Lemma 2.12$]$. If $\left(D_{1} \cdot C\right)=1$, then $\left.\pi\right|_{D_{1}}: D_{1} \rightarrow Y$ is a $\mathbb{P}^{r}$-subbundle. Therefore, condition (ii)(c) satisfied in the case $\operatorname{dim} \operatorname{Exc}(\pi)=\operatorname{dim} X, l(R)=r+2$, and $\left(D_{1} \cdot C\right)=1$.

Assume that $\pi$ is of fiber type, that $l(R)=r+2$, and that $\left(D_{1} \cdot C\right)=2$. Under the isomorphism $X \simeq \mathbb{P}_{Y}\left(\pi_{*} \mathcal{O}_{X}(L)\right)$, we have a natural exact sequence

$$
\left.0 \rightarrow \mathcal{O}_{\mathbb{P}}(1)\left(-D_{1}\right) \rightarrow \mathcal{O}_{\mathbb{P}}(1) \rightarrow \mathcal{O}_{\mathbb{P}}(1)\right|_{D_{1}} \rightarrow 0,
$$

where $\mathcal{O}_{\mathbb{P}}(1)$ is the tautological invertible sheaf on $\mathbb{P}_{Y}\left(\pi_{*} \mathcal{O}_{X}(L)\right)$. After taking $p_{*}$, we also obtain an exact sequence

$$
0 \rightarrow 0 \rightarrow \pi_{*} \mathcal{O}_{X}(L) \rightarrow\left(\left.p\right|_{D_{1}}\right)_{*}\left(\left.\mathcal{O}_{\mathbb{P}}(1)\right|_{D_{1}}\right) \rightarrow 0
$$

by cohomology and base change theorem, since $h^{i}\left(\mathbb{P}^{r+1}, \mathcal{O}(-1)\right)=0(i=0$, 1). Hence, condition (ii)(d) holds in the case $\operatorname{dim} \operatorname{Exc}(\pi)=\operatorname{dim} X, l(R)=$ $r+2$, and $\left(D_{1} \cdot C\right)=2$.

\subsection{Properties on scrolls}

In this section, we consider special toric varieties of rational scrolls. First, we fix notation. 
Notation 2.24. Let $s, t$ be positive integers, and let $a_{0}, \ldots, a_{t}$ be integers with $0=a_{0} \leq a_{1} \leq \cdots \leq a_{t}$. Let $X:=\mathbb{P}\left[\mathbb{P}^{s} ; a_{0}, \ldots, a_{t}\right]$; that is, $X=$ $\mathbb{P}_{\mathbb{P} s}\left(\mathcal{O}\left(a_{0}\right) \oplus \cdots \oplus \mathcal{O}\left(a_{t}\right)\right)$. Also let $D_{i}:=\mathbb{P}\left[\mathbb{P}^{s} ; a_{0}, \ldots, a_{i-1}, a_{i+1}, \ldots, a_{t}\right] \subset_{\text {can }}$ $X=\mathbb{P}\left[\mathbb{P}^{s} ; a_{0}, \ldots, a_{t}\right]$, that is, the canonical embedding, for any $0 \leq i \leq t$. (See Notation and terminology in Section 1.)

The following lemma is well known.

LEMMA 2.25. We have the following properties:

(1) $\operatorname{Pic}(X)=\mathbb{Z}[\mathcal{O}(1 ; 0)] \oplus \mathbb{Z}[\mathcal{O}(0 ; 1)]$;

(2) $\mathcal{O}_{X}\left(-K_{X}\right) \simeq \mathcal{O}\left(s+1-\sum_{i=1}^{t} a_{i} ; t+1\right)$;

(3) $D_{i} \in\left|\mathcal{O}\left(-a_{i} ; 1\right)\right|$ for any $0 \leq i \leq t$;

(4) $\operatorname{deg}_{C_{f}}\left(\left.\mathcal{O}(u ; v)\right|_{C_{f}}\right)=v$, and $\operatorname{deg}_{C_{h}}\left(\left.\mathcal{O}(u ; v)\right|_{C_{h}}\right)=u$, where $C_{f}$ is a line in a fiber of $X \rightarrow \mathbb{P}^{s}$ and $C_{h}$ is a line in $\mathbb{P}\left[\mathbb{P}^{s} ; a_{0}\right] \subset_{\text {can }} X=\mathbb{P}\left[\mathbb{P}^{s} ; a_{0}, \ldots, a_{t}\right]$;

(5) $\operatorname{Nef}(X)=\mathbb{R}_{\geq 0}[\mathcal{O}(1 ; 0)]+\mathbb{R}_{\geq 0}[\mathcal{O}(0 ; 1)]$, and $\operatorname{Eff}(X)=\mathbb{R}_{\geq 0}[\mathcal{O}(1 ; 0)]+$ $\mathbb{R}_{\geq 0}\left[\mathcal{O}\left(-a_{t} ; 1\right)\right]$

(6) for a divisor $D=\sum_{i=1}^{t} c_{i} D_{i}+d H$ with $c_{i}, d \in \mathbb{Z}$, where $H$ is the pullback of a hyperplane in $\mathbb{P}^{s}, h^{0}\left(X, \mathcal{O}_{X}(D)\right)$ is equal to

$$
\begin{gathered}
\#\left\{\left(P_{1}, \ldots, P_{s}, Q_{1}, \ldots, Q_{t}\right) \in \mathbb{Z}^{\oplus s+t} \mid Q_{j} \geq-c_{j}(1 \leq j \leq t),\right. \\
\left.\sum_{1 \leq j \leq t} Q_{j} \leq 0, P_{i} \geq 0 \quad(1 \leq i \leq s), \sum_{1 \leq i \leq s} P_{i}-\sum_{1 \leq j \leq t} a_{j} Q_{j} \leq d\right\}
\end{gathered}
$$

(7) if there exists $D \in|\mathcal{O}(k ; 1)|$ such that $k<-a_{t-1}$, then $\operatorname{Supp} D \supset D_{t}$;

(8) if a member $D \in|\mathcal{O}(k ; 2)|$ is reduced, then $k \geq-a_{t}-a_{t-1}$;

(9) assume that $a_{t-2}<a_{t}$; then any effective and reduced divisor $D$ on $X$ with $D \in\left|\mathcal{O}\left(-a_{t}-a_{t-1} ; 2\right)\right|$ decomposes into two irreducible components $D^{t}$ and $D^{t-1}$ such that $D^{t} \sim D_{t}$ and $D^{t-1} \sim D_{t-1}$ : here $D^{t}=D_{t}$ if $a_{t-1}<a_{t}$. Furthermore, there exists $\sigma \in \operatorname{Aut}\left(X / \mathbb{P}^{s}\right)$ such that $\sigma\left(D^{t}\right)=$ $D_{t}$ and $\sigma\left(D^{t-1}\right)=D_{t-1}$ hold.

Corollary 2.26. Let $D$ be a member of $D \in|\mathcal{O}(c ; d)|$ for some $d>0$. Assume that $(X, D)$ is a log Fano manifold. Set $r:=r(X, D)$, and set $\iota:=$ $\iota(X, D)$.

(1) If $\iota \geq t$, then $d=1, t=\iota$, and $s \geq \iota-1$. Furthermore, if $s=\iota-1$, then $a_{1}=\cdots=a_{\iota-1}=0$ and $c=-a_{\iota}$.

(2) If $r \geq t$ (hence, $\iota \geq t$ holds), $s=r$, and $r \geq 2$, then we have $r=\iota$ and either $\left(a_{1}, \ldots, a_{r-2}, a_{r-1}, c\right)=\left(0, \ldots, 0,0,1-a_{r}\right)$ or $\left(0, \ldots, 0,1,-a_{r}\right)$. 
Proof. By Lemma 2.25(2), $\mathcal{O}_{X}\left(-\left(K_{X}+D\right)\right) \simeq \mathcal{O}\left(s+1-\sum_{i=1}^{t} a_{i}-c ; t+\right.$ $1-d)$. Hence, $t+1-d \geq \iota$ by Lemma 2.25(4). Thus, $d=1$ and $t=\iota$ if $\iota \geq t$ holds (resp., $d=1$ and $t=\iota=r$ if $r \geq t$ holds). We also note that $s+1-\sum_{i=1}^{\iota} a_{i}-c$ is at least $\iota$ and is a positive multiple of $r$ and $c \geq-a_{\iota}$. Hence, $s \geq \iota-1+\sum_{i=1}^{\iota-1} a_{i} \geq \iota-1$.

(1) If $s=\iota-1$, then $\iota-\sum_{i=1}^{\iota} a_{i} \geq \iota+c \geq \iota-a_{\iota}$. Therefore, $\sum_{i=1}^{\iota-1} a_{i}=0$ and $c=-a_{\iota}$.

(2) If $s=r$ and $r \geq 2$, then $r+1-\sum_{i=1}^{r} a_{i}-c$ is divisible by $r$ and $\sum_{i=1}^{r} a_{i}+c \geq \sum_{i=1}^{r-1} a_{i} \geq 0$. Hence, $\sum_{i=1}^{r} a_{i}+c=1$. Therefore, either $\left(a_{1}, \ldots\right.$, $\left.a_{r-2}, a_{r-1}, c\right)=\left(0, \ldots, 0,0,1-a_{r}\right)$ or $\left(0, \ldots, 0,1,-a_{r}\right)$.

Corollary 2.27. Let $r:=t-1$ with $r \geq 2$, and let $D \in|\mathcal{O}(c ; d)|$ for some $d>0$. Assume that $(X, D)$ is a log Fano manifold with $r \mid r(X, D)$. Then $d=2$ and $s \geq r-1$. Furthermore, if $s=r-1$, then $a_{1}=\cdots=a_{r-1}=0$ and $c=-a_{r}-a_{r+1}$.

Proof. We repeat the argument for Corollary 2.26. By Lemma 2.25(2), $\mathcal{O}_{X}\left(-\left(K_{X}+D\right)\right) \simeq \mathcal{O}\left(s+1-\sum_{i=1}^{r+1} a_{i}-c ; r+2-d\right)$. Thus, we have $d=2$ since $r+2-d$ is a positive multiple of $r$ and $r \geq 2$. We also know that $s \geq$ $r-1+\sum_{i=1}^{r+1} a_{i}+c \geq r-1+\sum_{i=1}^{r-1} a_{i} \geq r-1$ by Lemma 2.25(8). Furthermore, if $s=r-1$, then $r-\sum_{i=1}^{r+1} a_{i} \geq r+c \geq r-a_{r}-a_{r+1}$.

\section{$\S 3$. Examples}

In this section, we give some examples of log Fano manifolds with large $\log$ Fano indices.

\subsection{Example of dimension $2 \iota-1$ and log Fano (pseudo)index $\iota$}

First, we consider case (1) in Corollary 2.26, which is the important example of $(2 \iota-1)$-dimensional log Fano manifolds with the log Fano (pseudo)index $\iota$ (see Theorem 4.3).

Example O. Let $\iota \geq 2$, let $m \geq 0$, let $X=\mathbb{P}\left[\mathbb{P}^{\iota-1} ; 0^{\iota}, m\right]$, and let $D \in$ $|\mathcal{O}(-m ; 1)|$. We know that $\mathcal{O}(1 ; 1)$ is ample and that $\mathcal{O}_{X}\left(-\left(K_{X}+D\right)\right) \simeq$ $\mathcal{O}(1 ; 1)^{\otimes \iota}$. If $m>0$, then $D$ is unique and $D=\mathbb{P}\left[\mathbb{P}^{\iota-1} ; 0^{\iota}\right] \subset_{\text {can }} X=\mathbb{P}\left[\mathbb{P}^{\iota-1} ; 0^{\iota}\right.$, $m]$ by Lemma 2.25(7). If $m=0$, then $X=\mathbb{P}^{\iota-1} \times \mathbb{P}^{\iota}$ and $D \in\left|\mathcal{O}_{\mathbb{P}^{\iota-1} \times \mathbb{P}^{\iota}}(0,1)\right|$. Hence, any member $D \in\left|\mathcal{O}_{\mathbb{P}^{\iota-1} \times \mathbb{P}^{\iota}}(0,1)\right|$ is always an irreducible smooth divisor, and we may assume that $D=\mathbb{P}\left[\mathbb{P}^{\iota-1} ; 0^{\iota}\right] \subset$ can $\mathbb{P}\left[\mathbb{P}^{\iota-1} ; 0^{\iota}, m\right]$. (We note that $\operatorname{dim}|D|=\iota$ if $m=0$.) Thus, $(X, D)$ is a $(2 \iota-1)$-dimensional log Fano manifold with $r(X, D)=\iota(X, D)=\iota$ for any $D \in|\mathcal{O}(-m ; 1)|$. 


\subsection{Examples of dimension $2 r$ and $\log$ Fano index $r$}

Next, we give examples of $2 r$-dimensional log Fano manifolds with the $\log$ Fano indices $r$ (see Theorem 4.5).

Example I. Let $X:=\mathrm{Bl}_{\mathbb{P}^{r-2}} \mathbb{P}^{2 r} \stackrel{\mathrm{Bl}}{\longrightarrow} \mathbb{P}^{2 r}$, that is, the blowup of $\mathbb{P}^{2 r}$ along an $(r-2)$-dimensional linear subspace $\mathbb{P}^{r-2}$, and let $E \subset X$ be the exceptional divisor. Take any $D \in\left|\mathrm{Bl}^{*} \mathcal{O}_{\mathbb{P}^{2 r}}(1) \otimes \mathcal{O}_{X}(-E)\right|$. We have $\operatorname{dim}|D|=r+1$, and any $D$ is the strict transform of a hyperplane in $\mathbb{P}^{2 r}$ containing the center of the blowup. The invertible sheaf $\mathcal{H}:=\mathrm{Bl}^{*} \mathcal{O}_{\mathbb{P}^{2 r}}(2) \otimes \mathcal{O}_{X}(-E)$ is ample. We know that $\mathcal{O}_{X}\left(-\left(K_{X}+D\right)\right) \simeq \mathcal{H}^{\otimes r}$. Thus, $(X, D)$ is a $2 r$-dimensional $\log$ Fano manifold with $r(X, D)=r$ for any $D \in\left|\mathrm{Bl}^{*} \mathcal{O}_{\mathbb{P}^{2 r}}(1) \otimes \mathcal{O}_{X}(-E)\right|$.

Example II. Let $X:=\mathbb{P}^{r-1} \times \mathbb{P}^{r+1}$, and let $D$ be an effective divisor on $X$ such that $D \in\left|\mathcal{O}_{\mathbb{P}^{r-1} \times \mathbb{P}^{r+1}}(0,2)\right|$. Then $\operatorname{dim}|D|=(r+2)(r+3) / 2-1$, and $D$ is an SNC divisor if and only if $D$ is the pullback of a smooth or reducible hyperquadric in $\mathbb{P}^{r+1}$. In particular, a general element in the linear system is an SNC divisor. Let $\mathcal{H}:=\mathcal{O}_{\mathbb{P}^{r-1} \times \mathbb{P}^{r+1}}(1,1)$. Then $\mathcal{H}$ is ample and $\mathcal{O}_{X}\left(-\left(K_{X}+D\right)\right) \simeq \mathcal{H}^{\otimes r}$. Thus, $(X, D)$ is a $2 r$-dimensional log Fano manifold with $r(X, D)=r$ for any SNC $D \in\left|\mathcal{O}_{\mathbb{P}^{r-1} \times \mathbb{P}^{r+1}}(0,2)\right|$.

Example III. Let $X:=\mathbb{P}\left[\mathbb{P}^{r-1} ; 0^{r}, m_{1}, m_{2}\right]$, with $0 \leq m_{1} \leq m_{2}$ and $1 \leq m_{2}$, and let $D \in\left|\mathcal{O}\left(-m_{1}-m_{2} ; 2\right)\right|$. All reduced elements in $|D|$ can be seen in the sum of $\mathbb{P}\left[\mathbb{P}^{r-1} ; 0^{r}, m_{1}\right]$ and $\mathbb{P}\left[\mathbb{P}^{r-1} ; 0^{r}, m_{2}\right] \subset_{\text {can }} X=\mathbb{P}\left[\mathbb{P}^{r-1} ; 0^{r}, m_{1}, m_{2}\right]$ by Lemma 2.25(9). We note that

$$
\operatorname{dim}|D|= \begin{cases}2 & \left(m_{1}=m_{2}\right), \\
\left(\begin{array}{c}
m_{2}+r-1 \\
r-1 \\
r-1
\end{array}\right)+r-1 & \left(m_{1}=0\right), \\
\left(\begin{array}{c}
m_{2}-m_{1}+r-1 \\
r-1
\end{array}\right) & \left(0<m_{1}<m_{2}\right),\end{cases}
$$

by Lemma 2.25(6). Let $\mathcal{H}:=\mathcal{O}(1 ; 1)$. Then $\mathcal{H}$ is ample and $\mathcal{O}_{X}\left(-\left(K_{X}+\right.\right.$ $D)) \simeq \mathcal{H}^{\otimes r}$. Thus, $(X, D)$ is a $2 r$-dimensional $\log$ Fano manifold with $r(X, D)=r$ for any reduced $D \in\left|\mathcal{O}\left(-m_{1}-m_{2} ; 2\right)\right|$.

Example IV (see also Remark 3.1). Let $E:=\mathbb{P}\left[\mathbb{P}^{r-1} ; 0^{r+1}\right] \subset_{\text {can }} X^{\prime}:=$ $\mathbb{P}\left[\mathbb{P}^{r-1} ; 0^{r+1}, m\right]$, with $m \geq 0$. We note that $E \simeq \mathbb{P}^{r-1} \times \mathbb{P}^{r}$. Consider a smooth divisor $B$ in $X^{\prime}$ with $B \in|\mathcal{O}(0 ; 2)|$ such that the intersection $B \cap E$ is also smooth. We note that $H^{0}\left(X^{\prime}, \mathcal{O}(0 ; 2)\right) \rightarrow H^{0}\left(E,\left.\mathcal{O}(0 ; 2)\right|_{E}\right)$ is surjective since $H^{1}\left(X^{\prime}, \mathcal{O}(0 ; 2)(-E)\right)=0$ by Kodaira's vanishing theorem. Hence, general $B \in|\mathcal{O}(0 ; 2)|$ satisfies this property. We note that

$$
\operatorname{dim}|\mathcal{O}(0 ; 2)|=\left(\begin{array}{c}
2 m+r-1 \\
r-1
\end{array}\right)+(r+1)\left(\begin{array}{c}
m+r-1 \\
r-1
\end{array}\right)+\frac{(r+1)(r+2)}{2}-1
$$


by Lemma 2.25(6). Let $\tau: X \rightarrow X^{\prime}$ be the double cover of $X^{\prime}$ with the branch divisor $B$, and let $D$ be the strict transform of $E$ on $X$. Then $X$ is smooth and $D \simeq \mathbb{P}^{r-1} \times \mathbb{Q}^{r}$ by construction. We know that $\mathcal{O}_{X}\left(-K_{X}\right) \simeq$ $\tau^{*}\left(\mathcal{O}_{X^{\prime}}\left(-K_{X^{\prime}}\right) \otimes \mathcal{O}(0 ;-1)\right) \simeq \tau^{*} \mathcal{O}(r-m ; r+2)$ and that $\mathcal{O}_{X}(D) \simeq \tau^{*} \mathcal{O}(-m$; 1). Let $\mathcal{H}:=\tau^{*} \mathcal{O}(1 ; 1)$, an ample invertible sheaf on $X$. Then $\mathcal{O}_{X}\left(-\left(K_{X}+\right.\right.$ $D)) \simeq \mathcal{H}^{\otimes r}$. We note that $\mathcal{H}$ cannot be divisible anymore by Remark 3.1. Thus, $(X, D)$ is a $2 r$-dimensional $\log$ Fano manifold with $r(X, D)=r$.

Example V (see also Remark 3.2). In this example, we consider the case $r \geq 3$. Let $D:=\mathbb{P}\left[\mathbb{Q}^{r} ; 0^{r}\right] \subset$ can $X:=\mathbb{P}\left[\mathbb{Q}^{r} ; 0^{r}, m\right]$, with $m \geq 0$. We note that $D \simeq \mathbb{P}^{r-1} \times \mathbb{Q}^{r}$. We also note that $\operatorname{dim}|D|=0$ if $m>0$. If $m=0$, then $X=\mathbb{P}^{r} \times \mathbb{Q}^{r}$ and $D \in\left|\mathcal{O}_{\mathbb{P}^{r} \times \mathbb{Q}^{r}}(1,0)\right|$; hence, $\operatorname{dim}|D|=r$ and $D$ is smooth. Let $\mathcal{H}:=\mathcal{O}(1 ; 1)$. Then $\mathcal{H}$ is ample and $\mathcal{O}_{X}\left(-\left(K_{X}+D\right)\right) \simeq \mathcal{H}^{\otimes r}$. Thus, $(X, D)$ is a $2 r$-dimensional $\log$ Fano manifold with $r(X, D)=r$.

Example VI. In this example, we consider only the case $r=2$. Let $D:=$ $\mathbb{P}_{\mathbb{P}^{1} \times \mathbb{P}^{1}}\left(\mathcal{O}^{\oplus 2}\right) \subset_{\text {can }} X:=\mathbb{P}_{\mathbb{P}^{1} \times \mathbb{P}^{1}}\left(\mathcal{O}^{\oplus 2} \oplus \mathcal{O}\left(m_{1}, m_{2}\right)\right)$, with $0 \leq m_{1} \leq m_{2}$. If $m_{2}>0$, then $\operatorname{dim}|D|=0$. If $m_{1}=m_{2}=0$, then $X=\mathbb{P}^{1} \times \mathbb{P}^{1} \times \mathbb{P}^{2}$ and $D \in\left|\mathcal{O}_{\mathbb{P}^{1} \times \mathbb{P}^{1} \times \mathbb{P}^{2}}(0,0,1)\right|$, and hence $\operatorname{dim}|D|=2$; any element in $|D|$ defines a smooth divisor. Let $\mathcal{H}:=p^{*} \mathcal{O}_{\mathbb{P}^{1} \times \mathbb{P}^{1}}(1,1) \otimes \mathcal{O}_{\mathbb{P}}(1)$, where $p: X \rightarrow \mathbb{P}^{1} \times \mathbb{P}^{1}$ is the projection and $\mathcal{O}_{\mathbb{P}}(1)$ is the tautological invertible sheaf with respect to the projection $p$. Then $\mathcal{H}$ is ample and $\mathcal{O}_{X}\left(-\left(K_{X}+D\right)\right) \simeq \mathcal{H}^{\otimes 2}$. Thus, $(X, D)$ is a 4-dimensional log Fano manifold with $r(X, D)=2$.

Example VII (see also Remark 3.3). Let $D:=\mathbb{P}_{\mathbb{P}^{r}}\left(T_{\mathbb{P}^{r}}\right) \subset_{\text {can }} X:=$ $\mathbb{P}_{\mathbb{P}^{r}}\left(T_{\mathbb{P}^{r}} \oplus \mathcal{O}(m)\right)$, with $m \geq 1$. If $m \geq 2$, then $\operatorname{dim}|D|=0$. If $m=1$, then $\operatorname{dim}|D|=r+1$. This follows from the exact sequence

$$
0 \rightarrow \mathcal{O}_{X} \rightarrow \mathcal{O}_{X}(D) \rightarrow \mathcal{N}_{D / X} \rightarrow 0
$$

and the fact that $\left.\mathcal{N}_{D / X} \simeq \mathcal{O}_{\mathbb{P}^{r} \times \mathbb{P}^{r}}(1-m, 1)\right|_{D}$ under an embedding $D \subset$ $\mathbb{P}^{r} \times \mathbb{P}^{r}$ of bidegree $(1,1)$. We note that there exists an embedding $X \subset$ $X_{1}:=\mathbb{P}\left[\mathbb{P}^{r} ; 1^{r+1}, m\right]$ obtained by the surjection $\alpha$ in the exact sequence

$$
0 \rightarrow \mathcal{O}_{\mathbb{P}^{r}} \rightarrow \mathcal{O}(1)^{\oplus r+1} \stackrel{\alpha}{\rightarrow} T_{\mathbb{P}^{r}} \rightarrow 0 .
$$

Let $\mathcal{H}:=\mathcal{O}(0 ; 1)$ on $X_{1}$. Then $\mathcal{H}$ is ample and $\left(\left.\mathcal{H}\right|_{X}\right)^{\otimes r} \simeq \mathcal{O}_{X}\left(-\left(K_{X}+D\right)\right)$. Thus, $(X, D)$ is a $2 r$-dimensional $\log$ Fano manifold with $r(X, D)=r$.

Example VIII. Let $X:=\mathbb{P}^{r} \times \mathbb{P}^{r}$, and let $D \in\left|\mathcal{O}_{\mathbb{P}^{r} \times \mathbb{P}^{r}}(1,1)\right|$. Then $\operatorname{dim}|D|=r(r+2)$, any smooth $D$ is isomorphic to $\mathbb{P}_{\mathbb{P}^{r}}\left(T_{\mathbb{P}^{r}}\right)$, and any nonsmooth $D$ is the union of the first and second pullbacks of hyperplanes. Let 
$\mathcal{H}:=\mathcal{O}_{\mathbb{P}^{r} \times \mathbb{P}^{r}}(1,1)$. Then $\mathcal{H}$ is ample and $\mathcal{O}_{X}\left(-\left(K_{X}+D\right)\right) \simeq \mathcal{H}^{\otimes r}$. Thus, $(X, D)$ is a $2 r$-dimensional log Fano manifold with $r(X, D)=r$ for any $D \in\left|\mathcal{O}_{\mathbb{P}^{r} \times \mathbb{P}^{r}}(1,1)\right|$.

Example IX. Let $X:=\mathbb{P}\left[\mathbb{P}^{r} ; 0^{r}, 1\right]$. We can view $X$ as the blowup of $P:=\mathbb{P}^{2 r}$ along an $(r-1)$-dimensional linear subspace $H \subset P$. Let $E$ be the exceptional divisor of the blowup. Then $E=\mathbb{P}\left[\mathbb{P}^{r} ; 0^{r}\right] \subset_{\text {can }} X=\mathbb{P}\left[\mathbb{P}^{r} ; 0^{r}, 1\right]$. Let $D \in|\mathcal{O}(0 ; 1)|$. Any smooth $D$ is the strict transform of a hyperplane in $P$ which does not contain $H$. Any nonsmooth $D$ can be written as $E+D_{0}$, where $D_{0}$ is the strict transform of a hyperplane in $P$ which contains $H$. Note that $\operatorname{dim}|D|=2 r$. Let $\mathcal{H}:=\mathcal{O}(1 ; 1)$. Then $\mathcal{H}$ is ample and $\mathcal{O}_{X}\left(-\left(K_{X}+D\right)\right) \simeq \mathcal{H}^{\otimes r}$. Thus, $(X, D)$ is a $2 r$-dimensional log Fano manifold with $r(X, D)=r$ for any $D \in|\mathcal{O}(0 ; 1)|$.

Example X. Let $X:=\mathbb{P}\left[\mathbb{P}^{r} ; 0^{r-1}, 1, m\right]$, with $m \geq 1$ and $D \in|\mathcal{O}(-m ; 1)|$. If $m \geq 2$, then $D$ is unique in $|\mathcal{O}(-m ; 1)|$ and $D=\mathbb{P}\left[\mathbb{P}^{r} ; 0^{r-1}, 1\right] \subset$ can $X=$ $\mathbb{P}\left[\mathbb{P}^{r} ; 0^{r-1}, 1, m\right]$. If $m=1$, then $\operatorname{dim}|D|=1$ by Lemma $2.25(6)$, and we may assume that $D=\mathbb{P}\left[\mathbb{P}^{r} ; 0^{r-1}, 1\right] \subset_{\text {can }} X=\mathbb{P}\left[\mathbb{P}^{r} ; 0^{r-1}, 1, m\right]$. In particular, any $D \in|\mathcal{O}(-m ; 1)|$ is a smooth divisor. Let $\mathcal{H}:=\mathcal{O}(1 ; 1)$. Then $\mathcal{H}$ is ample and $\mathcal{O}_{X}\left(-\left(K_{X}+D\right)\right) \simeq \mathcal{H}^{\otimes r}$. Thus, $(X, D)$ is a $2 r$-dimensional log Fano manifold with $r(X, D)=r$ for any $D \in|\mathcal{O}(-m ; 1)|$.

Example XI. Let $X:=\mathbb{P}\left[\mathbb{P}^{r} ; 0^{r}, m\right]$, with $m \geq 2$, and let $D \in \mid \mathcal{O}(-m+$ $1 ; 1) \mid$. We note that $\operatorname{Supp} D$ always contains $D_{0}:=\mathbb{P}\left[\mathbb{P}^{r} ; 0^{r}\right] \subset$ can $X=\mathbb{P}\left[\mathbb{P}^{r} ;\right.$ $\left.0^{r}, m\right]$ by Lemma $2.25(7)$. Furthermore, $D-D_{0}$ is the pullback of a hyperplane in $\mathbb{P}^{r}$ under the projection $p: X \rightarrow \mathbb{P}^{r}$. We also note that $\operatorname{dim} \mid \mathcal{O}(-m+$ $1 ; 1) \mid=r$ by Lemma $2.25(6)$. Let $\mathcal{H}:=\mathcal{O}(1 ; 1)$. Then $\mathcal{H}$ is ample and $\mathcal{O}_{X}\left(-\left(K_{X}+D\right)\right) \simeq \mathcal{H}^{\otimes r}$. Thus, $(X, D)$ is a $2 r$-dimensional log Fano manifold with $r(X, D)=r$ for any $D \in|\mathcal{O}(-m+1 ; 1)|$.

Now, we state some remarks about these examples.

Remark 3.1. In Example IV, the homomorphism $\tau^{*}: \operatorname{Pic}\left(X^{\prime}\right) \rightarrow \operatorname{Pic}(X)$ is an isomorphism. In particular, $\rho(X)=2$.

Proof. We can assume that $\mathbb{k}=\mathbb{C}$. If $m=0$, then $X^{\prime} \simeq \mathbb{P}^{r-1} \times \mathbb{P}^{r+1}$ and $X \simeq \mathbb{P}^{r-1} \times \mathbb{Q}^{r+1}$. Thus, the homomorphism $\tau^{*}$ is an isomorphism. We consider the case $m>0$. Let $R \subset X$ be the ramification divisor of $\tau$. We know that the linear system $|\mathcal{O}(0 ; 1)|$ on $X^{\prime}$ gives a divisorial contraction morphism $f: X^{\prime} \rightarrow Q$ contracting $E \simeq \mathbb{P}^{r-1} \times \mathbb{P}^{r}$ to $\mathbb{P}^{r}$. We note that $B \subset X^{\prime}$ is the pullback of some ample divisor $A \subset Q$. Thus, $H_{i}\left(\left(X^{\prime} \backslash B\right)^{\text {an }} ; \mathbb{Z}\right)=0$ for 
all $i>2 r+r-2$ by [GM, p. 25, (2.3) Theorem] for the proper morphism $\left.f\right|_{X^{\prime} \backslash B}: X^{\prime} \backslash B \rightarrow Q \backslash A$ to an affine variety. Thus, $H_{c}^{i}\left(\left(X^{\prime} \backslash B\right)^{\text {an }} ; \mathbb{Z}\right)=0$ for all $i<r+2$ by Poincaré's duality. We know that there exists an exact sequence

$$
H_{c}^{2}\left(\left(X^{\prime} \backslash B\right)^{\text {an }} ; \mathbb{Z}\right) \rightarrow H^{2}\left(\left(X^{\prime}\right)^{\text {an }} ; \mathbb{Z}\right) \stackrel{\alpha}{\rightarrow} H^{2}\left(B^{\text {an }} ; \mathbb{Z}\right) \rightarrow H_{c}^{3}\left(\left(X^{\prime} \backslash B\right)^{\text {an }} ; \mathbb{Z}\right) .
$$

Thus, $\alpha$ is an isomorphism. Applying the same argument to the composition $f \circ \tau: X \rightarrow Q$, we obtain the isomorphism $H^{2}\left(X^{\text {an }} ; \mathbb{Z}\right) \stackrel{\sim}{\rightarrow} H^{2}\left(R^{\text {an }} ; \mathbb{Z}\right) \simeq$ $H^{2}\left(B^{\text {an }} ; \mathbb{Z}\right)$. Therefore, $H^{2}\left(\left(X^{\prime}\right)^{\text {an }} ; \mathbb{Z}\right) \simeq H^{2}\left(X^{\text {an }} ; \mathbb{Z}\right)$. Therefore, $\operatorname{Pic}\left(X^{\prime}\right) \simeq$ $\operatorname{Pic}(X)$ by Lemma 2.12 .

Remark 3.2. If $m<0$, then $(X, D)$ in Example $\mathrm{V}$ is never a log Fano manifold.

Proof. Let $S:=\mathbb{P}\left[\mathbb{Q}^{r} ; m\right] \subset$ can $X=\mathbb{P}\left[\mathbb{Q}^{r} ; 0^{r}, m\right]$, the section of the projection $p: X \rightarrow \mathbb{Q}^{r}$. Then $\left.\mathcal{O}_{X}\left(-\left(K_{X}+D\right)\right)\right|_{S} \simeq \mathcal{O}_{\mathbb{Q}^{r}}(r(m+1))$. Therefore, $-\left(K_{X}+D\right)$ is never ample.

Remark 3.3. If $m<1$, then $(X, D)$ in Example VII is never a log Fano manifold.

Proof. Let $S:=\mathbb{P}_{\mathbb{P}^{r}}(\mathcal{O}(m)) \subset_{\text {can }} X=\mathbb{P}_{\mathbb{P}^{r}}\left(T_{\mathbb{P}^{r}} \oplus \mathcal{O}(m)\right)$. Then $\mathcal{O}_{X}\left(-\left(K_{X}+\right.\right.$ $D))\left.\right|_{S} \simeq \mathcal{O}_{\mathbb{P} r}(m r)$. Therefore, $-\left(K_{X}+D\right)$ is never ample.

Remark 3.4. In Examples I-XI, if $\left(X_{1}, D_{1}\right)$ and $\left(X_{2}, D_{2}\right)$ are from different examples, or are from the same example but their discrete parameters are not equal, then $X_{1} \neq X_{2}$. In particular, distinct $X$ s are nonisomorphic to each other except for those in Example IV.

\section{$\S 4$. Theorems}

In this section, we state the main classification results.

\subsection{Log Fano manifolds of del Pezzo type}

We classify $n$-dimensional log Fano manifolds $(X, D)$ with $r(X, D) \geq n-$ 1. The case $D=0$ is the well-known case of del Pezzo manifolds (see, e.g., [Ft2, I, Section 8]), hyperquadrics, and projective spaces (see [KO]). Hence, we consider the case $D \neq 0$. We note that the case $(n, r(X, D))=(2,1)$ has been treated by Maeda [M, Section 3]. We treat $\iota(X, D)$ instead of $r(X, D)$.

Proposition 4.1. Let $(X, D)$ be an $n$-dimensional log Fano manifold with $D \neq 0$. Then $\iota(X, D) \leq n$. If $\iota(X, D)=n$, then $X \simeq \mathbb{P}^{n}$ and $D \in|\mathcal{O}(1)|$ under this isomorphism. 
Proof. Assume that $\iota:=\iota(X, D) \geq n$. Choose an extremal ray $R$ such that $(D \cdot R)>0$. Let $\pi: X \rightarrow Y$ be the contraction morphism associated to $R$. Then $\operatorname{dim} \operatorname{Exc}(\pi)+\operatorname{dim} F \geq n+(\iota+1)-1 \geq 2 n$ for any nontrivial fiber $F$ of $\pi$ by Theorem 2.20 and Lemma 2.22. Hence, $\rho(X)=1, \iota=n$, and $-K_{X} \sim(n+1) D$. Then one can apply [KO, p. 32, Corollary].

Proposition 4.2. Let $(X, D)$ be an $n$-dimensional log Fano manifold with $n \geq 3, D \neq 0$, and $\iota(X, D)=n-1$. Then $X$ is isomorphic to $\mathbb{P}^{n}$ or $\mathbb{Q}^{n}$ unless $n=3$, and $(X, D)$ is isomorphic to the case $\iota=2$ in Example $O$ (see Section 3.1). Moreover, we have the following.

(1) If $X=\mathbb{P}^{n}$, then $D \in|\mathcal{O}(2)|$; that is, $D$ is a smooth or reducible hyperquadric.

(2) If $X=\mathbb{Q}^{n}$, then $D \in|\mathcal{O}(1)|$; that is, $D$ is a smooth hyperplane section.

Proof. Let $R$ be an extremal ray with a minimal rational curve $[C] \in R$ such that $(D \cdot R)>0$. Let $\pi: X \rightarrow Y$ be the contraction morphism associated to $R$. By Theorem 2.20, $\operatorname{dim} \operatorname{Exc}(\pi)+\operatorname{dim} F \geq n+l(R)-1 \geq 2 n-1$ holds for any nontrivial fiber $F$ of $\pi$. Hence, $\pi$ is of fiber type; that is, $X=\operatorname{Exc}(\pi)$ holds.

If $\rho(X)=1$, then $X$ itself is a Fano manifold and $\iota(X)>n-1$ by Proposition 2.13. If $\rho(X)=1$ and $\iota(X) \geq n+1$, then $X \simeq \mathbb{P}^{n}$ by [CMS] and $D \in|\mathcal{O}(2)|$. If $\rho(X)=1$ and $\iota(X)=n$, then $(D \cdot C)=1$ by Lemma 2.22 . Thus, $X \simeq \mathbb{Q}^{n}$ and $D \in|\mathcal{O}(1)|$ by [KO, p. 37, Corollary].

We consider the remaining case $\rho(X) \geq 2$. Then $\operatorname{dim} F=n-1$ for any fiber $F$ of $\pi$, and $l(R)=n$. Hence, $(D \cdot C)=1$ by Lemma 2.22. Therefore, $\pi$ is a $\mathbb{P}^{n-1}$-bundle over a smooth projective curve $Y$ by [Ft1, Theorem 2]. Then $Y \simeq \mathbb{P}^{1}$ since any extremal ray of $X$ is spanned by the class of a rational curve. We can assume that $X=\mathbb{P}\left[\mathbb{P}^{1} ; a_{0}, \ldots, a_{n-1}\right]$, where $0=a_{0} \leq$ $a_{1} \leq \cdots \leq a_{n-1}$. Thus, $1 \geq n-2$ by Corollary 2.26 . Since $n \geq 3$, we have $n=3, a_{1}=0$, and $D \in\left|\mathcal{O}\left(-a_{2} ; 1\right)\right|$ by Corollary $2.26(1)$. That is exactly the case which we have considered in Example $\mathrm{O}$ for the case $\iota=2$.

\subsection{Log Fano manifolds related to the Mukai conjecture}

We consider the log version of Wiśniewski's results ([W2], [W4]) related to the Mukai conjecture [Mu1, Conjecture 4]. These are the main results in this article.

TheOREM 4.3. Let $(X, D)$ be an $n$-dimensional log Fano manifold with $\iota:=\iota(X, D)>n / 2, D \neq 0$, and $\rho(X) \geq 2$. Then $n=2 \iota-1$, and $(X, D)$ is in Example $O$ in Section 3. 
Proof. We use induction on $n$. We may assume that $n \geq 5$ by Proposition 4.2. Choose an extremal ray $R$ with a minimal rational curve $[C] \in R$ as in Lemma 2.22, and let $\pi: X \rightarrow Y$ be the associated contraction. Then $l(R) \geq \iota+1 \geq 4$. We also choose an irreducible component with the conductor divisor $\left(D_{1}, E_{1}\right) \subset D$ such that $\left(D_{1} \cdot R\right)>0$. We know that $\rho\left(D_{1}\right) \geq 2$ by Lemma $2.21(2)$, and $\left(D_{1}, E_{1}\right)$ is an $(n-1)$-dimensional log Fano manifold with $\iota\left(D_{1}, E_{1}\right) \geq \iota, \rho\left(D_{1}\right) \geq 2$, and $\iota>n / 2>(n-1) / 2$. Hence, $E_{1}=0$ (hence, $D=D_{1}$ ) by induction step. Applying [NO, Theorem 3] to $D$, we have $n-1=2(\iota-1)$ and $D \simeq \mathbb{P}^{\iota-1} \times \mathbb{P}^{\iota-1}$. We know by Lemma 2.21 that $\left.\pi\right|_{D}$ contracts a curve. Since $D \simeq \mathbb{P}^{\iota-1} \times \mathbb{P}^{\iota-1},\left.\pi\right|_{D}: D \rightarrow \pi(D)$ is not birational. Thus, $\pi: X \rightarrow Y$ is of fiber type by Lemma 2.21, and $\left.\pi\right|_{D}: D \rightarrow Y$ is surjective since $(D \cdot R)>0$. We know that $\left.\pi\right|_{D}: D \rightarrow Y$ is an algebraic fiber space by Lemma 2.22. Hence, $\left.\pi\right|_{D}$ is isomorphic to the first projection $p_{1}: \mathbb{P}^{\iota-1} \times \mathbb{P}^{\iota-1} \rightarrow \mathbb{P}^{\iota-1}$. In particular, $\operatorname{dim}\left(\pi^{-1}(y) \cap D\right)=\iota-1$ for any closed point $y \in Y \simeq \mathbb{P}^{\iota-1}$. Therefore, $\pi: X \rightarrow Y$ is a $\mathbb{P}^{\iota}$-bundle and $\left.\pi\right|_{D}: D \rightarrow Y$ is a $\mathbb{P}^{\iota-1}$-subbundle by Proposition $2.23(\mathrm{i})$. Since $D \simeq \mathbb{P}^{\iota-1} \times \mathbb{P}^{\iota-1}$, there exists an integer $m \in \mathbb{Z}$ such that $\left(\left.\pi\right|_{D}\right)_{*} \mathcal{N}_{D / X} \simeq \mathcal{O}_{\mathbb{P} \iota-1}(-m)^{\oplus \iota}$ by Lemma 2.16 . We also know by Lemma 2.16 that $X \simeq \mathbb{P}_{\mathbb{P}^{\iota-1}}\left(\pi_{*} \mathcal{O}_{X}(D)\right)$ and that the embedding $D \subset X$ is induced by the surjection $\alpha$ in the natural exact sequence

$$
0 \rightarrow \mathcal{O}_{\mathbb{P}^{\iota-1}} \rightarrow \pi_{*} \mathcal{O}_{X}(D) \stackrel{\alpha}{\rightarrow}\left(\left.\pi\right|_{D}\right)_{*} \mathcal{N}_{D / X} \rightarrow 0 .
$$

Since $\iota-1=(n+1) / 2-1 \geq 2$, this sequence always splits. Hence, $\pi_{*} \mathcal{O}_{X}(D) \simeq$ $\mathcal{O}_{\mathbb{P} \iota-1} \oplus \mathcal{O}_{\mathbb{P} \iota-1}(-m)^{\oplus \iota}$, and $D \subset X$ is the canonical embedding obtained by the projection $\mathcal{O}_{\mathbb{P}^{\iota-1}} \oplus \mathcal{O}_{\mathbb{P}^{\iota-1}}(-m)^{\oplus \iota} \rightarrow \mathcal{O}_{\mathbb{P}^{\iota-1}}(-m)^{\oplus \iota}$. This case has been already considered in Corollary $2.26(1) ; m \geq 0$ holds. This is exactly the case which we have considered in Example O.

We recall Wiśniewski's classification result.

TheOrem 4.4 ([W4, p. 145, Theorem]). If $X$ is an $(2 r-1)$-dimensional Fano manifold with $r(X)=r$ and $\rho(X) \geq 2$, then $X \simeq \mathbb{P}^{r-1} \times \mathbb{Q}^{r}, \mathbb{P}_{\mathbb{P}^{r}}\left(T_{\mathbb{P}^{r}}\right)$, or $\mathbb{P}\left[\mathbb{P}^{r} ; 0^{r-1}, 1\right]$.

Using Theorems 4.3 and 4.4, we classify log Fano manifolds $(X, D)$ with $r(X, D)=r \geq 2, \operatorname{dim} X=2 r$, and $D \neq 0$. Note that the case $r=1$ has been treated by Maeda [M, Section 3].

Theorem 4.5 (Main Theorem). Let $(X, D)$ be a $2 r$-dimensional log Fano manifold with $r(X, D)=r \geq 2, D \neq 0$ and $\rho(X) \geq 2$. Then $(X, D)$ is in exactly one of Examples $I-X I$.

We prove Theorem 4.5 in Section 5. 


\subsection{Classification of Mukai-type log Fano manifolds}

As an immediate consequence, we can classify $n$-dimensional log Fano manifolds $(X, D)$ with $r(X, D) \geq n-2$. Those with $D=0$ are well known and are called Mukai manifolds (see [I], [MMu], [Mu2], [W1], [W2], [W4]).

Corollary 4.6. Let $(X, D)$ be an $n$-dimensional log Fano manifold with $D \neq 0$ and $r:=r(X, D) \geq n-2$.

(1) If $n \leq 3$, then $(X, D)$ belongs to one in the list of [M, Sections 6-9].

(2) If $n=4$ and $\rho(X) \geq 2$, then $r=2$ and $(X, D)$ belongs to one in the list of Theorem 4.5 .

(3) If $n \geq 5$ and $\rho(X) \geq 2$, then $n=5, r=2$, and $(X, D)$ belongs to the case in Example $O$.

(4) If $n \geq 4, \rho(X)=1$, and $r \geq n-1$, then $(X, D)$ belongs to one in the list of Propositions 4.1 and 4.2.

(5) If $n \geq 4, \rho(X)=1$, and $r=n-2$, then $(X, D)$ is one of the following: (i) $X \simeq \mathbb{P}^{n}$ and $D \in|\mathcal{O}(3)|$; (ii) $X \simeq \mathbb{Q}^{n}$ and $D \in|\mathcal{O}(2)|$; (iii) $X \simeq V_{d}$ and $D \in|\mathcal{O}(1)|$, with $1 \leq d \leq 5$, where $V_{d}$ is a del Pezzo manifold of degree $d$ in the sense of Takao Fujita [Ft2, Theorem 8.11(1)-(5)], and $\mathcal{O}(1)$ is the ample generator of $\operatorname{Pic}\left(V_{d}\right)$. (Conversely, general $D \in|\mathcal{O}(1)|$ in (5)(i)-(iii) are smooth. Hence, the cases (5)(i)-(iii) actually occur.)

\section{§5. Proof of Main Theorem 4.5}

Let $L$ be an ample divisor on $X$ such that $-\left(K_{X}+D\right) \sim r L$. Pick an extremal ray $R$ with a minimal rational curve $[C] \in R$ such that $(D \cdot R)>0$, and let $\pi: X \rightarrow Y$ be the associated contraction morphism. Let $\left(D_{1}, E_{1}\right) \subset$ $D$ be an irreducible component of $D$ with its conductor divisor such that $\left(D_{1} \cdot R\right)>0$. By the assumption that $\rho(X) \geq 2$ and Lemma 2.21(2), the morphism $\left.\pi\right|_{D_{1}}: D_{1} \rightarrow \pi\left(D_{1}\right)$ is not a finite morphism and $\rho\left(D_{1}\right) \geq 2$ holds. Since $\left(D_{1}, E_{1}\right)$ is a $(2 r-1)$-dimensional log Fano manifold with $r \mid r\left(D_{1}, E_{1}\right)$, the possibility of $\left.\pi\right|_{D_{1}}: D_{1} \rightarrow \pi\left(D_{1}\right)$ (which is an algebraic fiber space by Lemma 2.22) is isomorphic to exactly one of the following list by Theorems 4.3 and 4.4:

(1) $\mathbb{P}^{r-1} \times \mathbb{Q}^{r} \stackrel{p_{1}}{\rightarrow} \mathbb{P}^{r-1}$, where $E_{1}=0$;

(2) $\mathbb{P}\left[\mathbb{P}^{r-1} ; 0^{r}, m\right] \stackrel{p}{\rightarrow} \mathbb{P}^{r-1}$, where $E_{1} \in|\mathcal{O}(-m ; 1)|$ with $m \geq 0$;

(3) $\mathbb{P}^{r-1} \times \mathbb{Q}^{r} \stackrel{p_{2}}{\longrightarrow} \mathbb{Q}^{r}$, where $E_{1}=0$;

(4) $\mathbb{P}_{\mathbb{P}^{r}}\left(T_{\mathbb{P}^{r}}\right) \stackrel{p}{\rightarrow} \mathbb{P}^{r}$, where $E_{1}=0$;

(5) $\mathbb{P}\left[\mathbb{P}^{r} ; 0^{r-1}, 1\right] \stackrel{p}{\rightarrow} \mathbb{P}^{r}$, where $E_{1}=0$; 
(6) $\mathbb{P}^{r} \times \mathbb{P}^{r-1} \stackrel{p_{1}}{\longrightarrow} \mathbb{P}^{r}$, where $E_{1} \in\left|\mathcal{O}_{\mathbb{P}^{r} \times \mathbb{P}^{r-1}}(1,0)\right|$ (Theorem 4.3 with $m=0$ );

(7) $\mathbb{P}\left[\mathbb{P}^{r-1} ; 0^{r}, m\right] \stackrel{\phi}{\rightarrow} Z$, the divisorial contraction morphism contracting $E_{1} \simeq \mathbb{P}^{r-1} \times \mathbb{P}^{r-1}$ to $\mathbb{P}^{r-1}$, where $m>0 ;$

(8) $\mathrm{Bl}_{\mathbb{P}^{r-2}} \mathbb{P}^{2 r-1} \stackrel{\mathrm{Bl}}{\longrightarrow} \mathbb{P}^{2 r-1}$, the blowup of $\mathbb{P}^{2 r-1}$ along a linear subspace $\mathbb{P}^{r-2}$, where $E_{1}=0$.

Remark 5.1. For cases $(1),(2)$, and $(8), \operatorname{dim}\left(F \cap D_{1}\right)=r$ for any nontrivial fiber $F$ of $\pi$. For cases $(3)-(7), \operatorname{dim}\left(F \cap D_{1}\right)=r-1$ for any nontrivial fiber $F$ of $\pi$.

\subsection{Fiber type case}

Here, we consider the case where $\pi$ is of fiber type. Since $\operatorname{dim} F \geq l(R)-$ $1 \geq r \geq 2$ for any fiber $F$ of $\pi$, we have $\operatorname{dim} D_{1}>\operatorname{dim} Y$. Hence, $\left.\pi\right|_{D_{1}}$ is surjective and belongs to one of the cases (1)-(6). (We note that $\left.\pi\right|_{D_{1}}$ is an algebraic fiber space by Lemma 2.22.)

Cases (1) and (2). Since $\operatorname{dim}\left(\left.\pi\right|_{D_{1}}\right)^{-1}(y)=r$ for any $y \in Y \simeq \mathbb{P}^{r-1}$, one of (ii)(b), (ii)(c), or (ii)(d) in Proposition 2.23 holds.

Case (1) Since $\left.\pi\right|_{D}$ is a $\mathcal{Q}^{r}$-bundle, only cases (ii)(b) and (ii)(d) can occur.

First, we consider the case (ii)(b). Since $D \simeq \mathbb{P}^{r-1} \times \mathbb{Q}^{r},\left.\pi\right|_{D}$ is isomorphic to the first projection, and we can write $\mathcal{N}_{D / X} \simeq \mathcal{O}_{\mathbb{P}^{r-1} \times \mathbb{Q}^{r}}(-m, 1)$ for some integer $m \in \mathbb{Z}$. Then $\left(\left.\pi\right|_{D}\right)_{*} \mathcal{N}_{D / X} \simeq \mathcal{O}_{\mathbb{P}^{r-1}}(-m)^{\oplus r+2}$ by Lemma 2.19 , and the sequence

$$
0 \rightarrow \mathcal{O}_{\mathbb{P}^{r-1}} \rightarrow \pi_{*} \mathcal{O}_{X}(D) \stackrel{\alpha}{\rightarrow} \mathcal{O}_{\mathbb{P}^{r-1}}(-m)^{\oplus r+2} \rightarrow 0
$$

is exact. Furthermore, $X$ is obtained as a smooth divisor belonging to $\left|p^{*} \mathcal{O}_{\mathbb{P}^{r-1}}(s) \otimes \mathcal{O}_{\mathbb{P}}(2)\right|$ in $\mathbb{P}:=\mathbb{P}_{\mathbb{P}^{r-1}}\left(\pi_{*} \mathcal{O}_{X}(D)\right)$ for some $s \in \mathbb{Z}$, where $p: \mathbb{P} \rightarrow$ $\mathbb{P}^{r-1}$ is the projection and $D$ is the complete intersection of $X$ with $H:=$ $\mathbb{P}\left[\mathbb{P}^{r-1} ;(-m)^{r+2}\right]$ in $\mathbb{P}$. Here $H \subset \mathbb{P}$ is the subbundle of $p$ obtained by the surjection $\alpha$ in the above exact sequence, by Lemma 2.18. Under the isomorphism $H \simeq \mathbb{P}^{r-1} \times \mathbb{P}^{r+1}$, the divisor $D \simeq \mathbb{P}^{r-1} \times \mathbb{Q}^{r}$ belongs to $\mid \mathcal{O}_{\mathbb{P}^{r-1} \times \mathbb{P}^{r+1}}(s-$ $2 m, 2) \mid$. Thus, $s \geq 2 m$ since $h^{0}\left(\mathbb{P}^{r-1} \times \mathbb{P}^{r+1}, \mathcal{O}_{\mathbb{P}^{r-1} \times \mathbb{P}^{r+1}}(t, 2)\right)=0$ for any $t<0$. If $s>2 m$, then the restriction homomorphism $\operatorname{Pic}\left(\mathbb{P}^{r-1} \times \mathbb{P}^{r+1}\right) \rightarrow$ $\operatorname{Pic}(D)$ is an isomorphism by the Lefschetz theorem, and $\mathcal{O}_{D}\left(-K_{D}\right) \simeq$ $\left.\mathcal{O}_{\mathbb{P}^{r-1} \times \mathbb{P}^{r+1}}(r-(s-2 m), r)\right|_{D}$ by adjunction, but $-K_{D}$ is divisible by $r$, which leads to a contradiction. Thus, $s=2 m$.

Claim 5.2. We have that $m \geq 0$ holds.

Proof. We first consider the case $r=2$. Since $\rho(X)=2$, we can write $\mathrm{NE}(X)=R+R^{\prime}$ and let the contraction morphism associated to $R^{\prime}$ be 
$\pi^{\prime}: X \rightarrow Y^{\prime}$. We note that any nontrivial fiber $F^{\prime}$ of $\pi^{\prime}$ satisfies $\operatorname{dim} F^{\prime}=1$ since no curve in $F^{\prime}$ can be contracted by $\pi$. If $\left(D \cdot R^{\prime}\right)>0$, then any nontrivial fiber $F^{\prime}$ of $\pi^{\prime}$ satisfies $\operatorname{dim} F^{\prime} \geq 2$ by the same argument used in Proposition 2.23, which is a contradiction. If $\left(D \cdot R^{\prime}\right)<0$, then $\operatorname{Exc}\left(\pi^{\prime}\right) \subset D$. Hence, $m>0$. If $\left(D \cdot R^{\prime}\right)=0$, then $R^{\prime}$ is a $K_{X}$-negative extremal ray and $l\left(R^{\prime}\right) \geq 2$ by the same argument in Proposition 2.23. Hence, $\pi^{\prime}$ is of fiber type by Theorem 2.20 . Thus, $\left.\pi^{\prime}\right|_{D}$ is not a finite morphism since $\left(D \cdot R^{\prime}\right)=0$. Therefore, $m \geq 0$.

Now we consider the case $r \geq 3$. The above exact sequence always splits; hence, $H=\mathbb{P}\left[\mathbb{P}^{r-1} ;(-m)^{r+2}\right] \subset_{\text {can }} \mathbb{P}=\mathbb{P}\left[\mathbb{P}^{r-1} ;(-m)^{r+2}, 0\right]$. Assume that $m<0$ holds. The total coordinate ring of $\mathbb{P}$ is the $\mathbb{Z}^{\oplus 2}$-graded polynomial ring $\mathbb{k}\left[x_{0}, \ldots, x_{r-1}, y_{0}, y_{1}, \ldots, y_{r+2}\right]$ with the grading $\operatorname{deg} x_{i}=(1,0)(1 \leq i \leq$ $r-1), \operatorname{deg} y_{0}=(0,1), \operatorname{deg} y_{i}=(m, 1) \quad(1 \leq i \leq r+2) ; X$ is obtained by a graded equation of bidegree $(2 m, 1)$. Since $m<0$, any bidegree $(2 m, 1)$ polynomial is obtained by linear combinations of the elements in $\left\{y_{i} y_{j}\right\}_{1 \leq i \leq j \leq r+2}$. Then any divisor obtained by graded equations of bidegree $(2 m, 1)$ has singular points along the points defined by the graded equations $y_{1}=\cdots=$ $y_{r+2}=0$ by the Jacobian criterion. This is a contradiction since $X$ must be a smooth divisor. Therefore, $m \geq 0$.

Hence, the above exact sequence splits. We now normalize the bundle structures for simplicity. That is, we rewrite $H:=\mathbb{P}\left[\mathbb{P}^{r-1} ; 0^{r+2}\right] \subset$ can $\mathbb{P}:=$ $\mathbb{P}\left[\mathbb{P}^{r-1} ; 0^{r+2}, m\right]$ with $m \geq 0 ; X$ is a smooth divisor on $\mathbb{P}$ with $X \in|\mathcal{O}(0 ; 2)|$ and $D=X \cap H$, and $D$ is smooth. Since $H \simeq \mathbb{P}^{r-1} \times \mathbb{P}^{r+1}$ and $D \simeq \mathbb{P}^{r-1} \times$ $\mathbb{Q}^{r}$, we can take the pullback of a point $S\left(\simeq \mathbb{P}^{r-1}\right) \subset H \simeq \mathbb{P}^{r-1} \times \mathbb{P}^{r+1} \stackrel{p_{2}}{\longrightarrow}$ $\mathbb{P}^{r+1}$ in $\mathbb{P}^{r+1}$ such that $S \cap D=\emptyset$. We can assume that $S$ is the section of $p: \mathbb{P} \rightarrow \mathbb{P}^{r-1}$ obtained by the canonical first projection, that is, that $S=$ $\mathbb{P}\left[\mathbb{P}^{r-1} ; 0\right] \subset_{\text {can }} \mathbb{P}=\mathbb{P}\left[\mathbb{P}^{r-1} ; 0^{r+2}, m\right]$. Then the relative linear projection from $S$ over $\mathbb{P}^{r-1} \simeq Y$ yields a morphism $\sigma: \mathbb{P} \backslash S \rightarrow X^{\prime}:=\mathbb{P}\left[\mathbb{P}^{r-1} ; 0^{r+1}, m\right]$ over $\mathbb{P}^{r-1} \simeq Y$. The restriction of $\sigma$ to $X$ gives a double cover $\tau: X \rightarrow X^{\prime}$. The branch divisor $B \subset X^{\prime}$ of $\tau$ is smooth with $B \in|\mathcal{O}(0 ; 2)|$. Since the strict transform of the divisor $D^{\prime}:=\mathbb{P}\left[\mathbb{P}^{r-1} ; 0^{r+1}\right] \subset_{\text {can }} X^{\prime}=\mathbb{P}\left[\mathbb{P}^{r-1} ; 0^{r+1}, m\right]$ in $X^{\prime}$ is exactly $D$, the intersection $B \cap D^{\prime}$ is also smooth. This is Example IV.

Now, we consider the case (ii)(d). We write $\mathcal{E}:=\pi_{*} \mathcal{O}_{X}(L)$; then $X \simeq$ $\mathbb{P}_{\mathbb{P}^{r-1}}(\mathcal{E}) \stackrel{p}{\rightarrow} \mathbb{P}^{r-1}$. We can write $\left.\mathcal{O}_{\mathbb{P}}(1)\right|_{D} \simeq \mathcal{O}_{\mathbb{P}^{r-1} \times \mathbb{Q}^{r}}(-m, 1)$ for some integer $m \in \mathbb{Z}$, where $\mathcal{O}_{\mathbb{P}}(1)$ is the tautological invertible sheaf on $X$ with respect to the projection $p$. Hence, $\mathcal{E} \simeq\left(\left.p\right|_{D}\right)_{*}\left(\left.\mathcal{O}_{\mathbb{P}}(1)\right|_{D}\right) \simeq \mathcal{O}_{\mathbb{P}^{r-1}}(-m)^{\oplus r+2}$ 
by Lemma 2.19. Therefore, $X \simeq \mathbb{P}^{r-1} \times \mathbb{P}^{r+1}$, which is Example II. Thus, we have completed the distinction of case $(1)$.

Case (2) For convenience, let $m_{1}:=m$, where $m$ is the value in Case (2). Then only case (ii)(c) can occur since $\left.\pi\right|_{D_{1}}$ is a $\mathbb{P}^{r}$-bundle. We note that $D$ has two irreducible components $D_{1}$ and $D_{2}$ since $E_{1}$ is irreducible. We note that $\left(D_{2} \cdot R\right)>0$ since $\pi$ is of fiber type and $\left.\pi\right|_{E_{1}}$ is surjective. Hence, $\rho\left(D_{2}\right) \geq 2$ by the previous argument. Therefore, $\left.\pi\right|_{D_{2}}: D_{2} \rightarrow Y$ is isomorphic to $\mathbb{P}\left[\mathbb{P}^{r-1} ; 0^{r}, m_{2}\right] \stackrel{p}{\rightarrow} \mathbb{P}^{r-1}$ with $m_{2} \geq 0$. That is, $D_{2}$ also satisfies the hypothesis of case (2) by repeating the same argument. We can assume that $0 \leq m_{1} \leq m_{2}$. Under the isomorphism $D_{1} \simeq \mathbb{P}\left[\mathbb{P}^{r-1} ; 0^{r}, m_{1}\right]$, we can write $\mathcal{N}_{D_{1} / X} \simeq \mathcal{O}(u ; 1)$, with $u \in \mathbb{Z}$. We have $u=-m_{2}$ since $\left.\mathcal{N}_{D_{1} / X}\right|_{D_{1} \cap D_{2}} \simeq$ $\mathcal{N}_{D_{1} \cap D_{2} / D_{2}}$ and $\mathcal{N}_{D_{1} \cap D_{2} / D_{2}} \simeq \mathcal{O}_{\mathbb{P}^{r-1} \times \mathbb{P}^{r-1}}\left(-m_{2}, 1\right)$. Hence,

$$
\begin{aligned}
p_{*} \mathcal{N}_{D_{1} / X} & \simeq p_{*}\left(p^{*} \mathcal{O}_{\mathbb{P}^{r-1}}\left(-m_{2}\right) \otimes \mathcal{O}_{\mathbb{P}}(1)\right) \\
& \simeq \mathcal{O}_{\mathbb{P}^{r-1}}\left(-m_{2}\right)^{\oplus r} \oplus \mathcal{O}_{\mathbb{P}^{r-1}}\left(m_{1}-m_{2}\right)
\end{aligned}
$$

Thus, the exact sequence

$$
0 \rightarrow \mathcal{O}_{\mathbb{P}^{r-1}} \rightarrow \pi_{*} \mathcal{O}_{X}\left(D_{1}\right) \rightarrow \mathcal{O}_{\mathbb{P}^{r-1}}\left(-m_{2}\right)^{\oplus r} \oplus \mathcal{O}_{\mathbb{P}^{r-1}}\left(m_{1}-m_{2}\right) \rightarrow 0
$$

splits since $m_{1} \leq m_{2}$. Therefore, $X \simeq \mathbb{P}\left[\mathbb{P}^{r-1} ; 0^{r}, m_{1}, m_{2}\right]$, with $0 \leq m_{1} \leq m_{2}$. We note that $D \in\left|\mathcal{O}\left(-m_{1}-m_{2} ; 2\right)\right|$ by Corollary 2.27. This is Example III.

Cases (3)-(6) Next, we consider cases (3)-(6). Then $\operatorname{dim}\left(\left.\pi\right|_{D_{1}}\right)^{-1}(y)=$ $r-1$ for any closed point $y \in Y$. Hence, only case (i) in Proposition 2.23 occurs.

Case (3) In this case, $Y$ is isomorphic to $\mathbb{Q}^{r}$.

First, we consider the case $r=2$. We have that $\left.\pi\right|_{D}$ is isomorphic to $p_{23}: \mathbb{P}^{1} \times \mathbb{P}^{1} \times \mathbb{P}^{1} \rightarrow \mathbb{P}^{1} \times \mathbb{P}^{1}$, and we can write $\mathcal{N}_{D / X} \simeq \mathcal{O}_{\mathbb{P}^{1} \times \mathbb{P}^{1} \times \mathbb{P}^{1}}\left(1,-m_{1}\right.$, $\left.-m_{2}\right)$, with $m_{1}, m_{2} \in \mathbb{Z}$.

Claim 5.3. We have that $m_{1}, m_{2} \geq 0$.

Proof. It is enough to show that $m_{1} \geq 0$. Let $f=\{t\} \times \mathbb{P}^{1} \subset \mathbb{P}^{1} \times \mathbb{P}^{1} \simeq Y$ be a fiber of $p_{1}: \mathbb{P}^{1} \times \mathbb{P}^{1} \rightarrow \mathbb{P}^{1}$, where $t \in \mathbb{P}^{1}$. Let $X_{f}:=\pi^{-1}(f)$, and let $D_{f}:=$ $\pi^{-1}(f) \cap D$. Then $X_{f} \rightarrow f$ is a $\mathbb{P}^{2}$-bundle, $D_{f}$ is a smooth divisor in $X_{f}$ with $D_{f} \neq 0$, and $\left.\left.\mathcal{O}_{X_{f}}\left(-\left(K_{X_{f}}+D_{f}\right)\right) \simeq \mathcal{O}_{X}\left(-\left(K_{X}+D\right)\right)\right|_{X_{f}} \simeq \mathcal{O}_{X}(2 L)\right|_{X_{f}}$. Thus, $\left(X_{f}, D_{f}\right)$ is a $\log$ Fano manifold such that $r\left(X_{f}, D_{f}\right)$ is an even number and $\rho\left(X_{f}\right)=2$. Hence, $D_{f}=\mathbb{P}\left[\mathbb{P}^{1} ; 0^{2}\right]\left(\simeq \mathbb{P}^{1} \times \mathbb{P}^{1}\right) \subset{ }_{\text {can }} X_{f}=\mathbb{P}\left[\mathbb{P}^{1} ; 0^{2}, m\right]$ with $m \geq 0$ by Proposition 4.2. Thus, $\mathcal{N}_{D_{f} / X_{f}} \simeq \mathcal{O}_{\mathbb{P}^{1} \times \mathbb{P}^{1}}(1,-m)$. Since $\mathcal{N}_{D_{f} / X_{f}} \simeq$ $\left.\mathcal{N}_{D / X}\right|_{D_{f}} \simeq \mathcal{O}_{\mathbb{P}^{1} \times \mathbb{P}^{1}}\left(1,-m_{1}\right)$, we have $m_{1}=m \geq 0$. 
We know that $\left(p_{23}\right)_{*} \mathcal{N}_{D / X} \simeq \mathcal{O}_{\mathbb{P}^{1} \times \mathbb{P}^{1}}\left(-m_{1},-m_{2}\right)^{\oplus 2}$ by Lemma 2.19 . Hence, we can show that the exact sequence obtained by Lemma 2.16

$$
0 \rightarrow \mathcal{O}_{\mathbb{P}^{1} \times \mathbb{P}^{1}} \rightarrow \pi_{*} \mathcal{O}_{X}(D) \rightarrow \mathcal{O}_{\mathbb{P}^{1} \times \mathbb{P}^{1}}\left(-m_{1},-m_{2}\right)^{\oplus 2} \rightarrow 0
$$

splits. As a result, we are able to show that $D=\mathbb{P}_{\mathbb{P}^{1} \times \mathbb{P}^{1}}\left(\mathcal{O}_{\mathbb{P}^{1} \times \mathbb{P}^{1}}^{\oplus 2}\right) \subset_{\text {can }} X=$ $\mathbb{P}_{\mathbb{P}^{1} \times \mathbb{P}^{1}}\left(\mathcal{O}_{\mathbb{P}^{1} \times \mathbb{P}^{1}}^{\oplus 2} \oplus \mathcal{O}_{\mathbb{P}^{1} \times \mathbb{P}^{1}}\left(m_{1}, m_{2}\right)\right)$ with $0 \leq m_{1} \leq m_{2}$ by Lemma 2.16. This is Example VI.

We now consider the remaining case $r \geq 3$. We can write the normal sheaf $\mathcal{N}_{D / X} \simeq \mathcal{O}_{\mathbb{P}^{r-1} \times \mathbb{Q}^{r}}(1,-m)$ with $m \in \mathbb{Z}$. Then $\left(\left.\pi\right|_{D}\right)_{*} \mathcal{N}_{D / X} \simeq \mathcal{O}_{\mathbb{Q}^{r}}(-m)^{\oplus r}$ by Lemma 2.19 . Hence, we can see that the exact sequence obtained by Lemma 2.16

$$
0 \rightarrow \mathcal{O}_{\mathbb{Q}^{r}} \rightarrow \pi_{*} \mathcal{O}_{X}(D) \rightarrow \mathcal{O}_{\mathbb{Q}^{r}}(-m)^{\oplus r} \rightarrow 0
$$

splits. Hence, $D=\mathbb{P}\left[\mathbb{Q}^{r} ; 0^{r}\right] \subset_{\text {can }} X=\mathbb{P}\left[\mathbb{Q}^{r} ; 0^{r}, m\right]$ by Lemma 2.16. This is Example $\mathrm{V}$; the divisor $-\left(K_{X}+D\right)$ is ample if and only if $m \geq 0$ by Remark 3.2. Thus, we have completed the distinction of case (3).

Case (4) We can write $\left(\left.\pi\right|_{D}\right)_{*} \mathcal{N}_{D / X} \simeq T_{\mathbb{P} r} \otimes \mathcal{O}_{\mathbb{P} r}(-m)$ with $m \in \mathbb{Z}$ by Lemma 2.16. Hence, we get the exact sequence

$$
0 \rightarrow \mathcal{O}_{\mathbb{P}^{r}} \rightarrow \pi_{*} \mathcal{O}_{X}(D) \rightarrow T_{\mathbb{P}^{r}} \otimes \mathcal{O}_{\mathbb{P}^{r}}(-m) \rightarrow 0
$$

with surjectivity following from Lemma 2.16. It is well known that

$$
\operatorname{Ext}_{\mathbb{P}^{r}}^{1}\left(T_{\mathbb{P}^{r}} \otimes \mathcal{O}_{\mathbb{P}^{r}}(-m), \mathcal{O}_{\mathbb{P}^{r}}\right) \simeq \begin{cases}0 & (m \neq 0), \\ \mathbb{k} & (m=0) .\end{cases}
$$

We also know that all nonsplit exact sequences for the case $m=0$ are obtained by

$$
0 \rightarrow \mathcal{O}_{\mathbb{P} r} \rightarrow \mathcal{O}_{\mathbb{P} r}(1)^{\oplus r+1} \rightarrow T_{\mathbb{P} r} \rightarrow 0
$$

If the exact sequence is not split, then $X \simeq \mathbb{P}^{r} \times \mathbb{P}^{r}$ by the above argument. This case has been considered in Example VIII. If the exact sequence splits, then we can show that $D=\mathbb{P}_{\mathbb{P}^{r}}\left(T_{\mathbb{P}^{r}}\right) \subset_{\text {can }} X=\mathbb{P}_{\mathbb{P}^{r}}\left(T_{\mathbb{P}^{r}} \oplus \mathcal{O}_{\mathbb{P}^{r}}(m)\right)$. This case has been considered in Example VII; the divisor $-\left(K_{X}+D\right)$ is ample if and only if $m \geq 1$ by Remark 3.3.

Case (5) We can write $\left(\left.\pi\right|_{D}\right)_{*} \mathcal{N}_{D / X} \simeq\left(\mathcal{O}_{\mathbb{P}^{r}}^{\oplus r-1} \oplus \mathcal{O}_{\mathbb{P}^{r}}(1)\right) \otimes \mathcal{O}_{\mathbb{P}^{r}}(-m)$ with $m \in \mathbb{Z}$ by Lemma 2.16. Since $r \geq 2$, the exact sequence

$$
0 \rightarrow \mathcal{O}_{\mathbb{P}^{r}} \rightarrow \pi_{*} \mathcal{O}_{X}(D) \rightarrow\left(\mathcal{O}_{\mathbb{P}^{r}}^{\oplus r-1} \oplus \mathcal{O}_{\mathbb{P}^{r}}(1)\right) \otimes \mathcal{O}_{\mathbb{P}^{r}}(-m) \rightarrow 0
$$


splits. Thus, $X \simeq \mathbb{P}\left[\mathbb{P}^{r} ; 0^{r-1}, 1, m\right]$ and $D \in|\mathcal{O}(-m ; 1)|$. Since $\mathcal{O}_{X}\left(-K_{X}\right) \simeq$ $\mathcal{O}(r-m ; r+1)$, we have $\mathcal{O}_{X}(L) \simeq \mathcal{O}(1 ; 1)$. We know from Corollary 2.26(2) that $m \geq 0$; this case has been considered in Examples IX and X.

Case (6) We can write $\left(\left.\pi\right|_{D_{1}}\right)_{*} \mathcal{N}_{D_{1} / X} \simeq \mathcal{O}_{\mathbb{P} r}(-m)^{\oplus r}$ with $m \in \mathbb{Z}$ by Lemma 2.16. Since $r \geq 2$, the exact sequence

$$
0 \rightarrow \mathcal{O}_{\mathbb{P}^{r}} \rightarrow \pi_{*} \mathcal{O}_{X}(D) \rightarrow \mathcal{O}_{\mathbb{P}^{r}}(-m)^{\oplus r} \rightarrow 0
$$

splits. Thus, $X \simeq \mathbb{P}\left[\mathbb{P}^{r} ; 0^{r}, m\right]$. We know from Corollary $2.26(2)$ that $m \geq 0$ holds; this case has been considered in Examples VIII, IX, and XI.

\subsection{Birational type case}

Here, we consider the case where $\pi$ is birational. We know that $\left.\pi\right|_{D_{1}}: D_{1} \rightarrow$ $\pi\left(D_{1}\right)$ is a birational morphism by Lemma $2.21(1)$ and an algebraic fiber space by Lemma 2.22 . Hence, $\left.\pi\right|_{D_{1}}: D_{1} \rightarrow \pi\left(D_{1}\right)$ belongs to case $(7)$ or case (8). However, we have $\operatorname{dim}\left(D_{1} \cap F\right)=r-1$ for any nontrivial fiber $F$ of $\pi$ for case (7); this contradicts Proposition 2.23(i). For case (8), we have $\operatorname{dim}(D \cap F)=r$ for any nontrivial fiber $F$ of $\pi$. Thus, only case(ii)(a) in Proposition 2.23 occurs. That is, $Y$ is smooth and $\pi$ is the blowup along a smooth projective subvariety $W \subset Y$ of dimension $r-2$. Let $D_{Y}:=\pi(D) \subset$ $Y$. Then $D_{Y} \simeq \mathbb{P}^{2 r-1}$, and $W \subset D_{Y}$ is a linear subspace of dimension $r-2$ under the isomorphism $D_{Y} \simeq \mathbb{P}^{2 r-1}$. Let $E \subset X$ be the exceptional divisor of $\pi$. Then $\pi^{*} D_{Y}=D+E$. We note that there exists a divisor $L_{Y}$ on $Y$ such that $\pi^{*} \mathcal{O}_{Y}\left(L_{Y}\right) \simeq \mathcal{O}_{X}(L+E)$ by Theorem 2.11 since $(E \cdot C)=-1$ and $(L \cdot C)=1$. Therefore, $\mathcal{O}_{Y}\left(r L_{Y}\right) \simeq \mathcal{O}_{Y}\left(-\left(K_{Y}+D_{Y}\right)\right)$ by Theorem 2.11 since $\pi^{*} \mathcal{O}_{Y}\left(r L_{Y}\right) \simeq \mathcal{O}_{X}(r L+r E) \simeq \mathcal{O}_{X}\left(-\left(K_{X}+D\right)+r E\right) \simeq \mathcal{O}_{X}\left(-\pi^{*} K_{Y}-D-\right.$ $E) \simeq \pi^{*} \mathcal{O}_{Y}\left(-\left(K_{Y}+D_{Y}\right)\right)$.

Claim 5.4. We have that $\left(Y, D_{Y}\right)$ is also a log Fano manifold with $r$ $r\left(Y, D_{Y}\right)$.

Proof. It is enough to show that $L_{Y}$ is an ample divisor on $Y$. We know that $\mathrm{NE}(Y)$ is a closed convex cone since $\mathrm{NE}(X)$ is. Hence, it is enough to show that $\left(L_{Y} \cdot C_{Y}\right)>0$ for any irreducible curve $C_{Y} \subset Y$. If $C_{Y} \not \subset W$, taking the strict transform $\widehat{C}_{Y}$ of $C_{Y}$ in $X$, then $\left(L_{Y} \cdot C_{Y}\right)=\left(L \cdot \widehat{C}_{Y}\right)+\left(E \cdot \widehat{C}_{Y}\right)>0$. Hence, it is enough to treat the case $C_{Y} \subset W$. We note that $W \subset D_{Y}$ and all curves in $D_{Y}$ are numerically proportional since $D_{Y} \simeq \mathbb{P}^{2 r-1}$. Therefore, we can reduce to the case $C_{Y} \not \subset W$.

Since $D_{Y} \simeq \mathbb{P}^{2 r-1}$, we have $\rho(Y)=1$ by Lemma $2.21(2)$. Thus, $Y \simeq \mathbb{P}^{2 r}$ and $D_{Y} \in|\mathcal{O}(1)|$ by [Ft2, Theorem 7.18]. This is Example I. 
Therefore, we have completed the proof of Theorem 4.5.

Acknowledgments. The author is grateful to János Kollár for showing him an earlier version of [Kol3] and for making a suggestion to classify SNC Fano varieties. He also would like to express his gratitude to Shigefumi Mori, Noboru Nakayama, and Daniel Allcock for making various suggestions. He thanks Eiichi Sato and Kazunori Yasutake for suggesting that he replace $r(X, D)$ with $\iota(X, D)$ in Theorem 4.3.

\section{REFERENCES}

[A] F. Ambro, Quasi-log varieties (in Russian), Tr. Mat. Inst. Steklova 240 (2003), 220-239; English translation in Proc. Steklov Inst. Math. 240 (2003), 214-233. MR 1993751.

[ABW] M. Andreatta, E. Ballico, and J. A. Wiśniewski, Two theorems on elementary contractions, Math. Ann. 297 (1993), 191-198. MR 1241801. DOI 10.1007/ BF01459496.

[AW] M. Andreatta and J. A. Wiśniewski, A note on nonvanishing and applications, Duke. Math. J. 72 (1993), 739-755. MR 1253623. DOI 10.1215/ S0012-7094-93-07228-6.

[CMS] K. Cho, Y. Miyaoka, and N. I. Shepherd-Barron, "Characterizations of projective spaces and applications to complex symplectic manifolds" in Higher Dimensional Birational Geometry (Kyoto, 1997), Adv. Stud. Pure Math. 35, Math. Soc. Japan, Tokyo, 2002, 1-88. MR 1929792.

[F] O. Fujino, Introduction to the log minimal model program for log canonical pairs, preprint, arXiv:0907.1506v1 [math.AG]

[Ft1] T. Fujita, "On polarized manifolds whose adjoint bundles are not semipositive" in Algebraic Geometry (Sendai, 1985), Adv. Stud. Pure Math. 10, North-Holland, Amsterdam, 1987, 167-178. MR 0946238.

[Ft2] - Classification Theories of Polarized Varieties, London Math. Soc. Lecture Note Ser. 155, Cambridge University Press, Cambridge, 1990. MR 1162108. DOI 10.1017/CBO9780511662638.

[GM] M. Goresky and R. MacPherson, Stratified Morse Theory, Ergeb. Math. Grenzgeb. (3) 14, Springer, Berlin, 1988. MR 0932724.

[I] V. A. Iskovskikh, Fano threefolds, I, Izv. Ross. Akad. Nauk Ser. Mat. 41 (1977), 516-562, 717; II, 42 (1978), 506-549. MR 0463151.

[KO] S. Kobayashi and T. Ochiai, Characterizations of complex projective spaces and hyperquadrics, J. Math. Kyoto Univ. 13 (1973), 31-47. MR 0316745.

[Kol1] J. Kollár, "Adjunction and discrepancies" in Flips and Abundance for Algebraic Threefolds (Salt Lake City, 1991), Astérisque 211, Soc. Math. France, Paris, 1992, 183-192. MR 1225842.

[Kol2] - Singularities of the Minimal Model Program, Cambridge Tracts in Math. 200, Cambridge University Press, Cambridge, 2013. MR 3057950.

[Kol3] , New examples of terminal and log canonical singularities, preprint, arXiv:1107.2864v1 [math.AG] 
[KolM] J. Kollár and S. Mori, Birational Geometry of Algebraic Varieties, Cambridge Tracts in Math. 134, Cambridge University Press, Cambridge, 1998. MR 1658959. DOI 10.1017/CBO9780511662560.

[M] H. Maeda, Classification of logarithmic Fano threefolds, Compos. Math. 57 (1986), 81-125. MR 0817298.

[Mat] H. Matsumura, Commutative Ring Theory, Cambridge Stud. Adv. Math. 8, Cambridge University Press, Cambridge, 1986. MR 0879273.

$[\mathrm{MMu}] \mathrm{S}$. Mori and S. Mukai, Classification of Fano 3-folds with $B_{2} \geq 2$, Manuscripta Math. 36 (1981/82), 147-162; Erratum, Manuscripta Math. 110 (2003), 407. MR 0641971. DOI 10.1007/BF01170131.

[Mu1] S. Mukai, "Problems on characterization of the complex projective space" in Birational Geometry of Algebraic Varieties, Open Problems (Katata, 1988), Taniguchi Foundation, Katata, 1988, 57-60.

[Mu2] - Biregular classification of Fano 3-folds and Fano manifolds of coindex 3, Proc. Natl. Acad. Sci. USA 86 (1989), 3000-3002. MR 0995400. DOI 10.1073/ pnas.86.9.3000.

[NO] C. Novelli and G. Occhetta, Rational curves and bounds on the Picard number of Fano manifolds, Geom. Dedicata 147 (2010), 207-217. MR 2660578. DOI 10. 1007/s10711-009-9452-4.

[W1] J. Wiśniewski, Fano 4-folds of index 2 with $b_{2} \geq 2$ : A contribution to Mukai classification, Bull. Pol. Acad. Sci. Math. 38 (1990), 173-184. MR 1194261.

[W2] - On a conjecture of Mukai, Manuscripta Math. 68 (1990), 135-141. MR 1063222. DOI 10.1007/BF02568756.

[W3] - On contractions of extremal rays of Fano manifolds, J. Reine Angew. Math. 417 (1991), 141-157. MR 1103910. DOI 10.1515/crll.1991.417.141.

[W4] - On Fano manifolds of large index, Manuscripta Math. 70 (1991), 145152. MR 1085628. DOI 10.1007/BF02568366.

Research Institute for Mathematical Sciences

Kyoto University

Kyoto 606-8502

Japan

fujita@kurims.kyoto-u.ac.jp 